\title{
The chip-flow behaviors and formation mechanisms in the orthogonal cutting process of Ti6Al4V alloy
}

\author{
Wei Ma*, Xiangyu Chen, Fei Shuang \\ Institute of Mechanics, Chinese Academy of Sciences, Beijing 100190, China
}

\section{A R T I C L E I N F O}

\section{Keywords:}

Metal orthogonal cutting

Chip flow stability

Plastic instability

Shear deformation localization

Instability criterion

\begin{abstract}
A B S T R A C T
This work involves experimental and analytical investigations of chip flow stability in metal cutting process. First, in cutting experiments of Ti6Al4V alloy, the transformation of chip morphology from continuous to serrated and later to discontinuous was observed as the cutting speed increased. Scanning electron microscopic (SEM) observation of the shear fracture surface demonstrated shear-localized instability and intergranular failure behaviors. Then we used the improved orthogonal cutting model (OCM) to analyze the plastic flow process of work materials in a plane strain state. A corresponding governing equation system was set up, the dimensionless governing parameters were determined by dimensional analysis, and an instability criterion was established by linear perturbation analysis. Analytical results showed that the plastic instability of chip flow could take place in a continuous chip, which is different from the shear-localized instability in a serrated chip. Finally, in terms of the balance conditions between the kinetic energy and the surface energy, the sawtooth growth behavior in serrated chips and the formation mechanism of discontinuous chips were studied.
\end{abstract}

\section{Introduction}

The high-speed machining (HSM) process plays a crucial role in the rapidly developing manufacturing industry. Due to the nonlinear and dynamic nature of the process, technological improvement and processing optimization require a better understanding of its physical essence. Generally, the process involves plastic instability, rate sensitivity, friction heating, material softening and convection. For such a complex process, a great number of accomplishments have been achieved due to continuous improvement in theoretical models. However, evident discrepancies still appear between analytical results and experimental observations, arising from the unrealistic assumptions adopted in the models. Hence, a pressing research need is to further improve current models for the analysis of the HSM process.

The orthogonal cutting model (OCM) is widely used since it describes the main features of metal cutting. This model supposes that the ratio of workpiece width to chip thickness is large enough for edge effects to be neglected, and the work-material deformation is treated as a plane strain state. The cut depth $h_{1}$, rake angle $\alpha_{T}$ or shear angle $\varphi$, and the cutting speed $V_{0}$ determine the cutting conditions (Fig. 1). The one-dimensional OCMs in common use, such as the shear plane model (Merchant, 1945) (Fig. 1a) and the card model (Piispanen, 1948) (Fig. 1b) assumed that the chip velocity on the shear plane $o A$ is discontinuous. However, the experimental observation (Palmer and Oxley, 1959) and the analysis of the slip-line field of chip flow (Enahoro and Oxley, 1966) show that the chip develops in a finite thickness zone: the primary shear zone (PSZ), in which the material experiences very high rates of shear. The parallel-sided shear zone model used by Oxley and Welsh (1963) considered a PSZ thickness that was

\footnotetext{
* Corresponding author.

E-mail address: watwm@imech.ac.cn (W. Ma).
} 

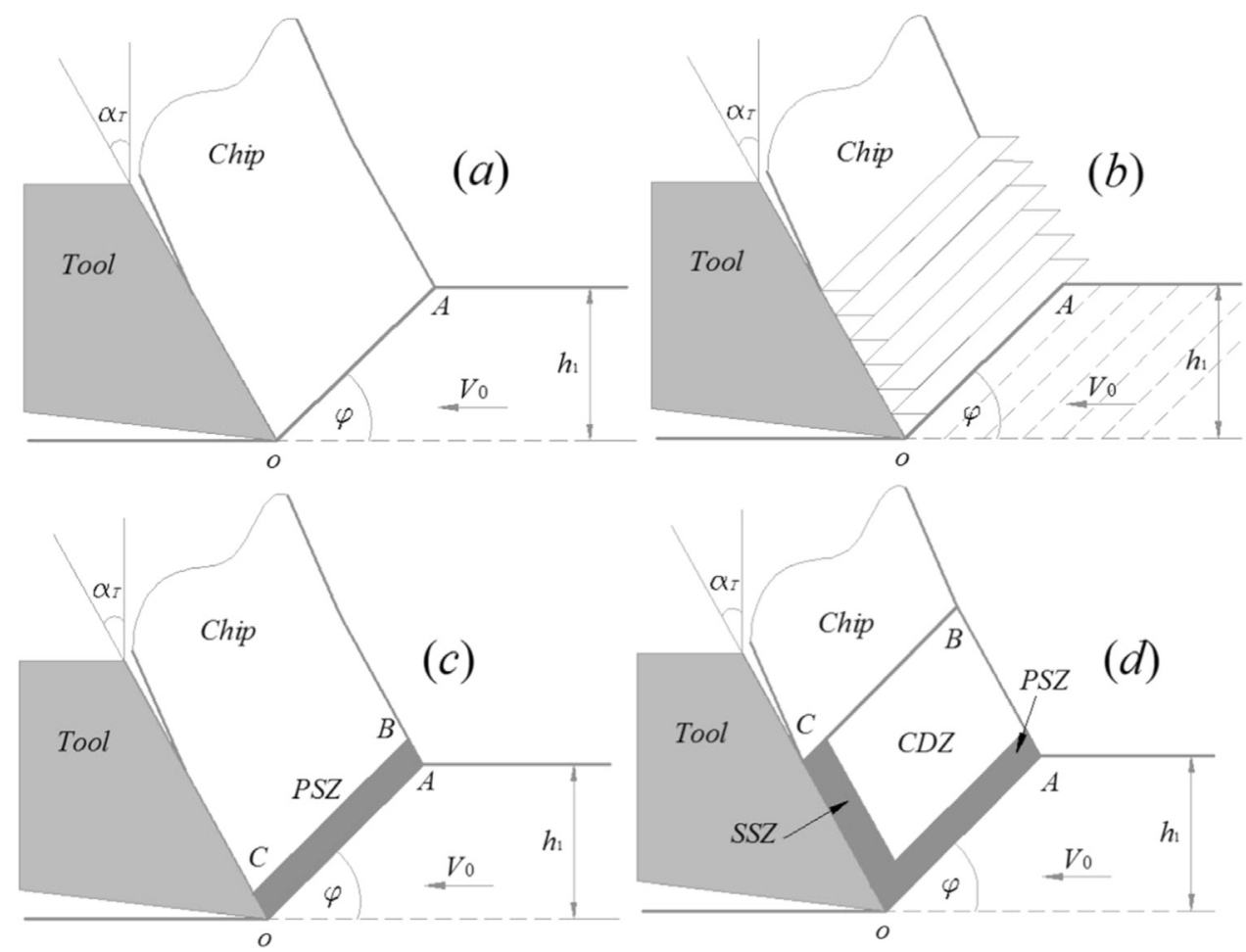

Fig. 1. Different OCMs used for the studies of metallic cutting behaviors: (a) the shear plane model (Merchant, 1945), (b) the card model (Piispanen, 1948), (c) the parallel-sided zone model (Oxley and Welsh, 1963), and (d) the expanding chip formation zone model (Stevenson and Oxley, 1970-1971).

quite small (Fig. 1c). Hence, this model describes not only the continuous change in the chip velocity but also the effects of the strain rate and the temperature of work-material. As such, the one-dimensional models (Fig. 1a-c) mainly consider the effects of shearlocalized deformation on serrated chip formation. The two-dimensional model involves the chip flow in the expanding chip formation zone $O A B C$ that contains the PSZ and the secondary shear zone (SSZ) (Fig. 1d). In terms of the slip-line field OCM, Stevenson and Oxley (1970-1971) studied the effect of friction on stress distribution at the tool-chip interface, and Fang (2003) studied the size effects of tool edge roundness and the shear zone. In the present work, we will use the model to study the plastic flow process and the formation mechanism of chips in a plane strain state.

So far, the OCMs have been used to study the formation mechanisms of chips with different shapes, for example, the continuous chip formed in the low-speed cutting process, and the serrated and discontinuous chips in the HSM process. Advances have also been achieved in research on the machining of new materials, particularly for the hard-to-cut Ti6Al4V and Inconel 718 alloys. Accordingly, two distinguishing theories on serrated chip formation have been developed on the basis of OCMs. The first one involves the shear band instability during serrated chip formation. In the model of Burns and Davies (1997), the normal pressure on the tool rake face is introduced, and the Hopf bifurcation theory is used to explain the transition from the continuous chip to the serrated one. The model of Ye et al. (2013) is based on the momentum diffusion theory of Grady and Kipp (1987). Molinari et al. (2002), by using the OCM, studied the dependence of serration frequency on cutting speeds in serrated chips; recently, through dimensional analysis and numerical simulations, the authors uncovered the coupled effects of rate sensitivity, thermal softening, inertia, thermal conduction, and convection on the metallic cutting mechanism (Molinari et al., 2013). Ma et al. (2012), using a plane strain OCM, determined the instability condition of chip flow. Komanduri and Huo (2001) carried out a thermal analysis of metal cutting. The heating of work material comes from the shear-localized deformation on the shear plane and the friction at the tool-chip interface.

The second theory is based on fracture mechanics, and it is supported by a large number of experimental observations. Taylor (1906) first observed the crack, called as the Taylor crack, propagating along the cutting direction in the ductile metal cutting process. Shaw et al. (1991) ascribed the serrated chip formation to the cyclic cracking of material along a shear plane. Elbestawi et al. (1996) took for granted the chip formation as a propagation process of mixed-model cracks and predicted the direction of crack initiation and propagation using the energy criterion. Poulachon and Moisan (1998) showed that the cracks propagating along the shear plane in hardened steel result in serrated chip formation. Barry and Byrne (2002) attributed the shear banding instability of the PSZ material to cyclic cracking and thermal softening. Atkins (2003) applied the ductile fracture mechanics in the modeling of the metal cutting process and revealed the dependence of shear angles on the fracture toughness and the dissipation rate of energy. $\mathrm{Gu}$ et al. (2013) showed that the adiabatic shear-localized fracture leads to the formation of serrated chips or discontinuous chips. The numerical simulation of Chen et al. (2011) on the Ti6AL4V alloy cutting was also based on the ductile fracture model.

Recently, the formation mechanism of serrated chips has attracted growing attention due to its close relationship with tool life 
and the integrity of machining surface. To characterize the serrated chip shape geometrically, Molinari et al. (2013) defined the segmentation index, Sutter and List (2013) defined the degree of segmentation, and Atlati et al. (2011) defined the segmentation intensity ratio. These scholars showed that the frequency of chip segmentation is related to the cutting speed. Cotterell and Byrne (2008) examined the geometrical features of single chip segmentation and determined the critical strain of shear-localized instability by studying the sawtooth cycle and frequency. Barry et al. (2001) investigated the acoustic emission phenomenon in Ti6Al4V alloy machining and asserted that the serrated chip arose from thermal plastic instability and ductile fracture. These theoretical predictions and modeling results have been confirmed in the machining experiments of Ti6Al4V alloy (Gente and Hoffmeister, 2001; Bäker et al., 2002).

From the above reviews, we can see that the current analyses focused mainly on the shear-localized instability of material in the PSZ. The serrated chip morphology is described in terms of geometric parameters or via direct comparison between experimental observations and simulation results. Mathematical description, in combination with the OCM, leads normally to a one-dimensional partial differential equation. Little attention is paid to the two-dimensional flow process of chip materials, as Sekhon and Chenot (1993) and Ma et al. (2012) did. At present, in-depth investigations of the chip flow in plane strain state and sawtooth growth mechanisms in serrated chip formation are still lacking.

These topics are addressed in the present work. In the cutting process of metals, work material first separates at the tool edge, and then the chip and machined workpiece move along the tool rake face and the cutting direction, respectively. Convection has a strong effect on chip formation and removal. In order to consider the convection effect, we propose a two-dimensional continuum model. The purpose is not only to characterize the development of shear bands in serrated chip formation, but also to describe the stability of two-dimensional chip flows in the expanding chip formation zone.

In this study, we first conducted high-speed machining experiments of Ti6Al4V alloy and then performed post-mortem microscopic observations on recovered chips. Theoretically, we first used a semi-analytic method to construct an approximate flow field in the chip formation zone, then applied a linear perturbation method to perform a linear stability analysis for chip flows, and finally, based on the principle of energy balance, analyzed the discontinuous chip formation.

The intention of the present study is threefold: (i) to reveal the basic mechanism of chip formation and removal through highspeed cutting experiments on Ti6Al4V alloy, (ii) to develop a two-dimensional analytical model to describe the plastic flow of chips with different shapes, and (iii) to provide a better understanding of the stability of chip plastic flows in metal cutting.

\section{Machining experiments}

\subsection{Procedure description}

Currently, we use high-rate loading technologies on the basis of light-gas guns and Hopkinson bar apparatuses to perform machining experiments on Ti6Al4V alloy. Fig. 2 illustrates the arrangement of the machining apparatus, including the machining assembly and a 60-mm bore laboratory compression-shear gas gun. The apparatus is capable of providing sufficient high-impact momentum to model the HSM process. The largest cutting speed approaches $300 \mathrm{~m} / \mathrm{s}$ for the projectile assembly of $1 \mathrm{~kg}$, which consists of a specimen, a specimen holder, and a Bakelite projectile. The employment of large mass-projectile assemblages ensures that the machining process is stationary over the cutting distance of $40 \mathrm{~mm}$. The cutting speeds depend on the pressure of the air gun, the mass of the projectile assembly, and the distance between the launch tube exit and the site of the projectile in the launch tube. In the current machining tests, a wide range of cutting speeds, from 0.05 to $210 \mathrm{~m} / \mathrm{s}$, is achieved. Because the rotation of projectile assemblages at the acceleration stage in the tube is constrained, the cut depth can be precisely controlled; e.g., the maximum relative error is within the limit of $10 \%$, as the cut depth is larger than $100 \mu \mathrm{m}$. To study the influence of the cut depth on the removal of materials, we may conduct a certain kind of machining experiment with the same cutting speed and preset cut depths by adjusting the set of micrometer calipers. In addition to orthogonal machining experiments, we can conduct oblique machining experiments with this apparatus. As shown in the inset of Fig. $2 \mathrm{a}$, when the tool edge is set to a normal cutting velocity (i.e., $\theta=0$ ), the cutting experiment models the orthogonal cutting process; otherwise, it simulates the oblique cutting process as $\theta \neq 0$.

In the present study, we adopted the experimental approach of Sutter et al. (1997), that is, the tools are fixed on the holding fixtures while the specimen moves with the projectile assembly in the tube. This design is convenient for measuring the temperature and vibration of tools and for collecting the chips in the chip pool (Fig. 2b) for the post-mortem microscopic observations of the failure mechanism of chip materials. We may also fix the specimen on the holding fixtures and let the tool move in the launch tube if our focus is on the plastic deformation and the temperature change of the workpiece. The machining experiments with a cutting speed in the range of $20-210 \mathrm{~m} / \mathrm{s}$ were completed using the light-gas gun setup. When the cutting speed was in the range of 5$20 \mathrm{~m} / \mathrm{s}$, we replaced the light-gas gun by a Hopkinson pressure bar system. The low-speed cutting experiments from 0.05 to $5 \mathrm{~m} / \mathrm{s}$ were conducted on a CK6150B computer-controlled lathe. The workpiece material was Ti-6Al-4V alloy, of which the mechanical properties and constitutive parameters are given in Table 1. An uncoated P10 carbide tool was used in these machining processes. The details of the machining experiments can be found in Ye et al. (2012).

\subsection{Results and discussion}

Fig. 3a-f show scanning electron microscopic (SEM) observations of the longitudinal midsections of the chips generated at the same magnifications and at various cutting speeds, from 0.05 to $60 \mathrm{~m} / \mathrm{s}$. We can see that, at low cutting speeds $\left(V_{0}<0.5 \mathrm{~m} / \mathrm{s}\right)$ (Fig. $3 a$ ), continuous chips form. In the cutting speed range of $0.5-60 \mathrm{~m} / \mathrm{s}$, serrated chips develop (Fig. $3 b-f$ ). Clearly, the serrated 

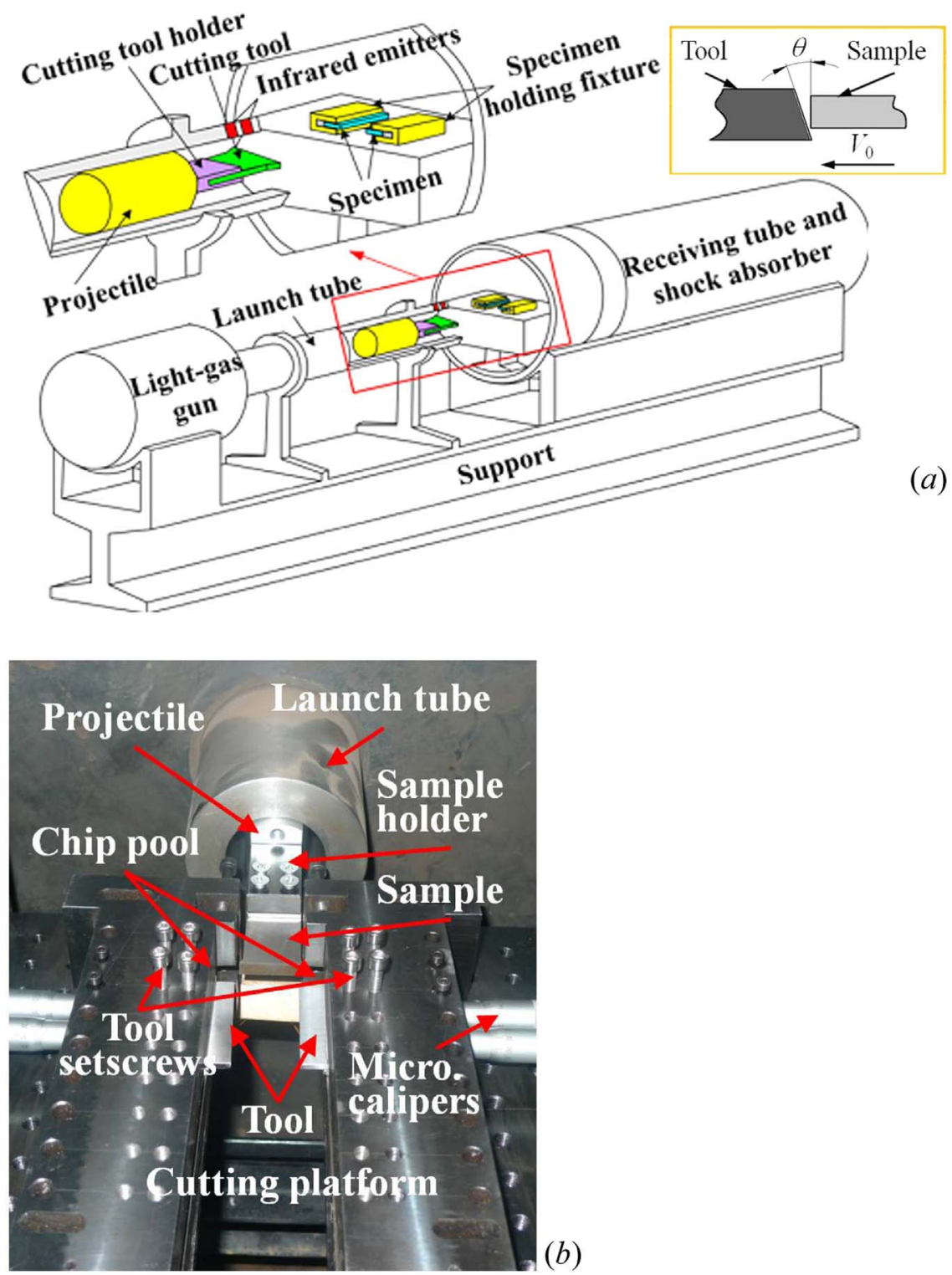

(b)

Fig. 2. (a) Schematic of the light gas gun testing setup for modeling the orthogonal cutting process of materials at high cutting speeds (Ye et al., 2012). The inset shows the schematic plot for the oblique cutting process; $(b)$ the real photograph of the cutting platform of materials.

Table 1

The mechanical properties and Johnson-Cook (J-C) constitutive parameters of Ti6Al4V alloy (Lee and Lin, 1998).

\begin{tabular}{lll}
\hline Material parameters, symbol and unit & & Values \\
\hline Mass density $\rho\left(\mathrm{kg} / \mathrm{m}^{3}\right)$ & & 4420 \\
Thermal conductivity $\lambda\left(\mathrm{W} /\left(\mathrm{m}^{\circ} \mathrm{C}\right)\right)$ & & 7.2 \\
Specific heat capacity c $\left(\mathrm{J} /\left(\mathrm{kg}^{\circ} \mathrm{C}\right)\right)$ & & \\
Elastic modulus $E(\mathrm{GPa})$ & & 114 \\
Poisson's ratio $v$ & & 0.342 \\
Melting temperature $T_{m}\left({ }^{\circ} \mathrm{C}\right)$ & & $1930 \mathrm{~K}$ \\
Shear flow stress $(\mathrm{MPa})$ & $A_{0}(\mathrm{MPa})$ & 498 \\
Shear yield strain & $B_{0}(\mathrm{MPa})$ & 0.2 \\
$\mathrm{~J}-\mathrm{C}$ model parameters & $C_{0}$ & 782.7 \\
& $n$ & 498.4 \\
& $m$ & 0.028 \\
& & 0.28 \\
\end{tabular}



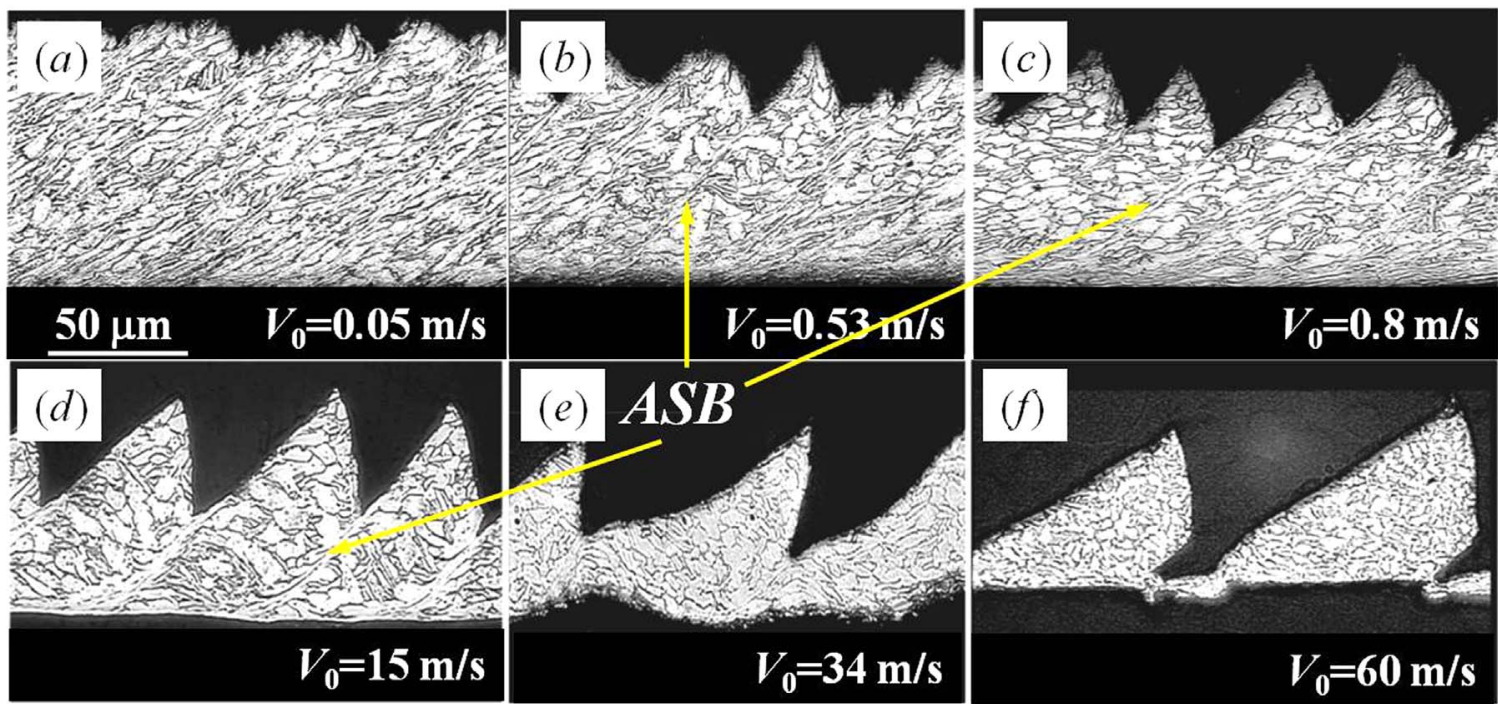

Fig. 3. SEM photographs of serrated chips of Ti6Al4V alloy in the orthogonal cutting process at different cutting speeds (Ye et al., 2012).

chips result from the adiabatic shear bands in the PSZ. The cutting speed $V_{0}=0.5 \mathrm{~m} / \mathrm{s}$ denotes the transition threshold for the chip from a continuous to a serrated shape. The microstructures of the shear deformation zone and the free surface of chips have been studied in the previous work of the authors (Ye et al., 2012). They showed that the shear plastic flow induces an elongated dimple microstructure and crystalline refinement. The free surface exhibits a relatively fine lamellar structure, as observed by Barry et al. (2001) and Wang et al. (2011). The details are not restated here; readers can refer to the Ref. (Ye et al., 2012).

Now our attention turns to microstructural observations of the shear-localized fracture surface of the discontinuous chip. The experimental results in Fig. 3 show that, when the cutting speed approaches $60 \mathrm{~m} / \mathrm{s}$, the contact length between the segmentations tends toward zero, and that discontinuous chips consisting of isolated debris develop. Fig. $4 a-c$ show the macrographs of the discontinuous chips obtained in the high-speed cutting experiments. We can see that the thickness of discontinuous chips gradually decreases with increasing cutting speeds. The result indicates that the shear band spacing in the Ti6Al4V alloy chip decreases with increasing cutting speed. This tendency is in agreement with the theoretical predictions of Wright and Ockendon (1996) and Molinari (1997).

To gain an insight into the mechanisms of discontinuous chip formation, the microstructures of the shear-localized fracture surface of the chip were inspected via SEM. Fig. 5 shows the shear fracture surfaces on which we performed SEM observations. Fig. 6 shows enlarged SEM views of the shear fracture surface, on which the sites where the SEM micrographs in Figs. 7 and 8 were taken from are illustrated.
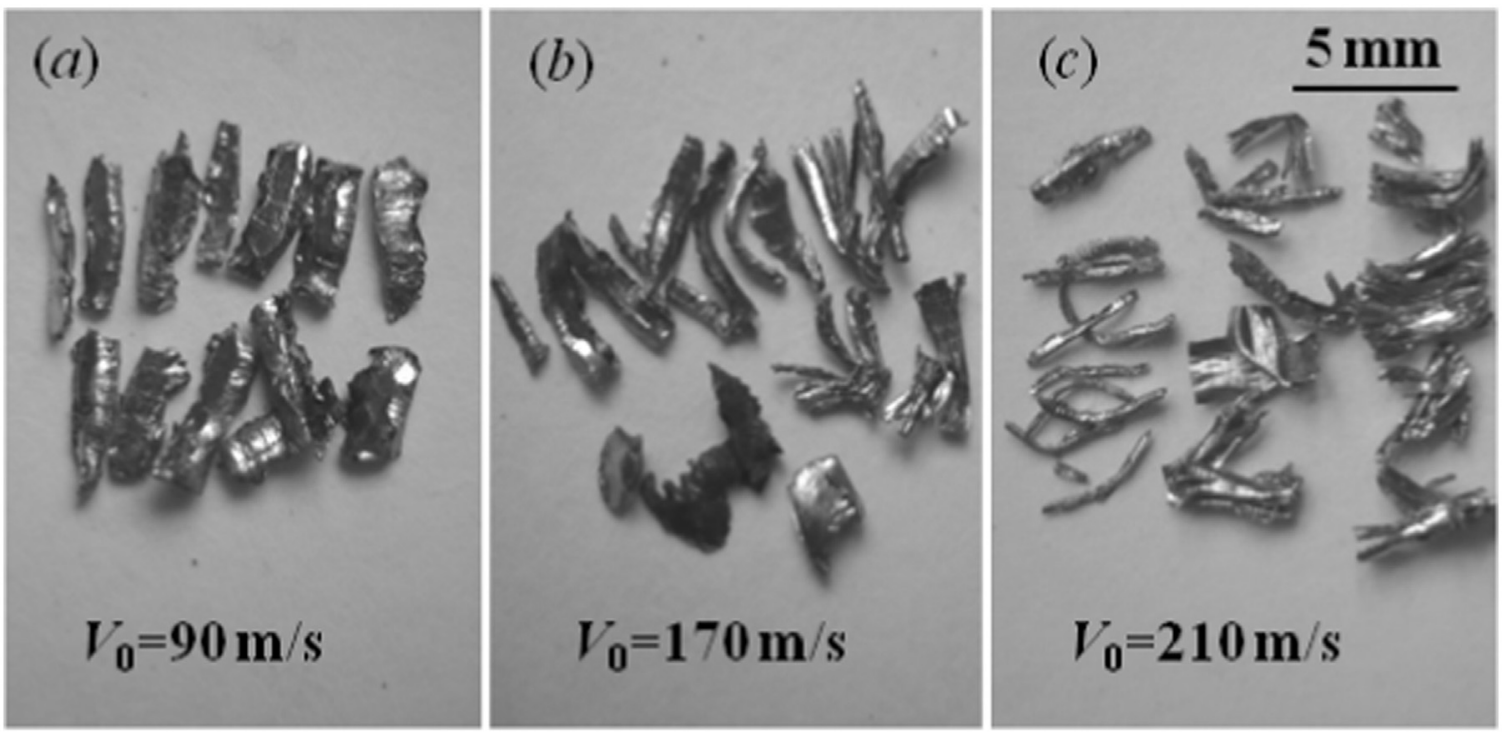

Fig. 4. Macrographs of discontinuous chips of Ti6Al4V alloy in the orthogonal cutting process at high cutting speeds (a) $90 \mathrm{~m} / \mathrm{s}$, (b) $170 \mathrm{~m} / \mathrm{s}$, and (c) $210 \mathrm{~m} / \mathrm{s}$. 


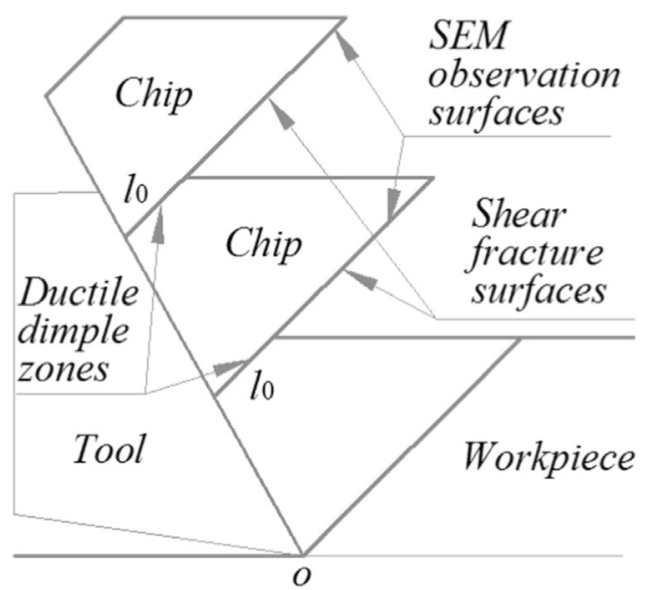

Fig. 5. Schematic diagram of serrated chip formation in the HSM. When the length of shear-localized deformation zone goes to zero $\left(l_{0} \rightarrow 0\right)$, the serrated chip changes into a discontinuous chip. The SEM photographs of Figs. 6-8 are taken from the shear fracture surfaces as indicated by the arrows in the figures

The experimental results in Fig. 3 show the shape change of the serrated chips as the cutting speed increased, which actually demonstrates the formation process of discontinuous chips. Thus, we identify that the discontinuous chip was the result of further development of the serrated chip under high-speed cutting conditions. The main mechanism was the shear-localized instability of chip materials in the PSZ following the shear fracture. The former did not produce new surface on the chip free surface but the latter did. We can see that each shear fracture surface has two distinct microstructural zones. One is the common ductile dimple structure zone with a lesser area. The dimple zone appears near the margin of the shear fracture surface, which implies that the dimple
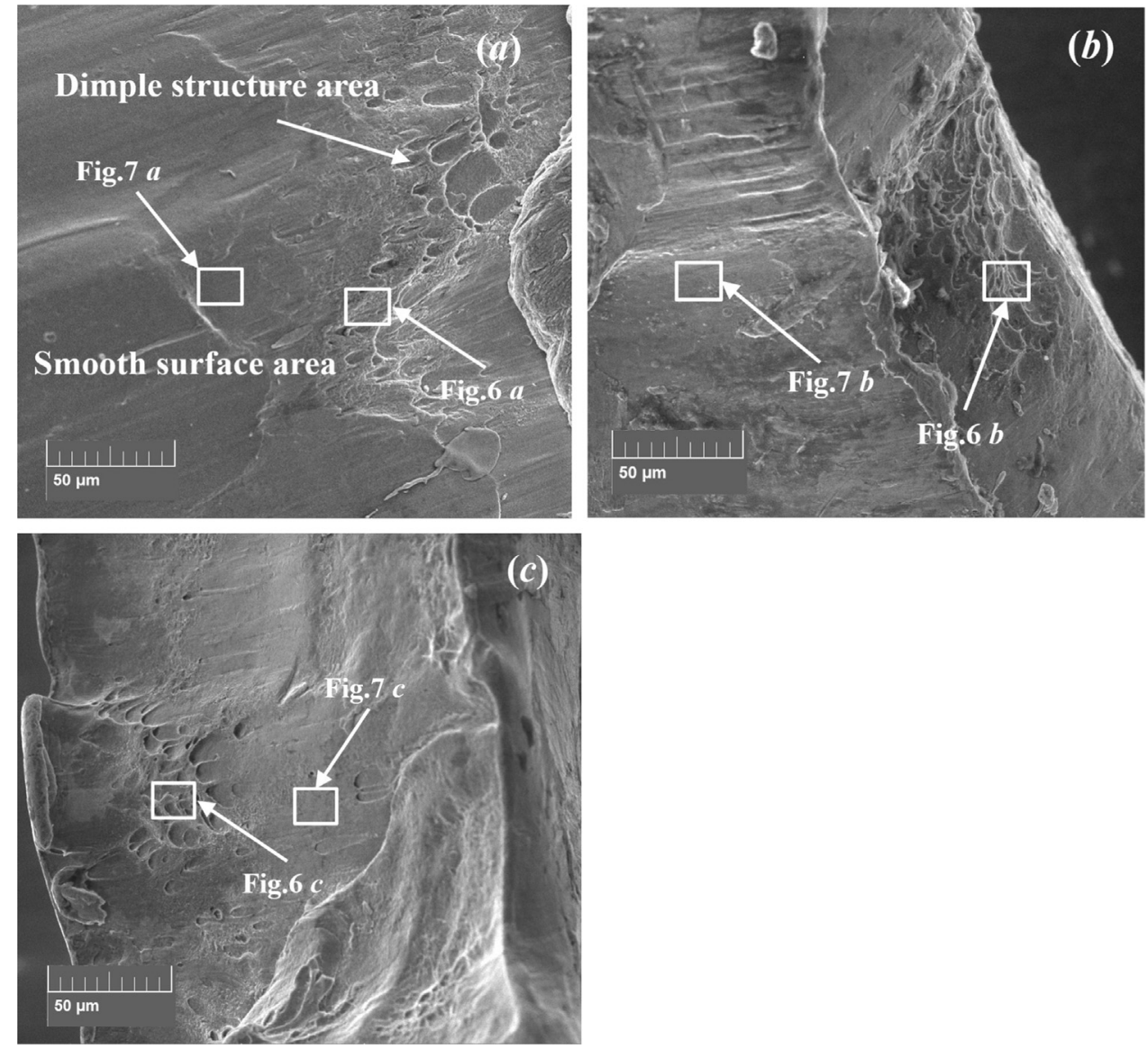

Fig. 6. SEM views of the fracture surfaces in the shear-localized deformation region in the Ti6Al4V alloy HSM process. The cutting speeds are equal to $(a) 90 \mathrm{~m} / \mathrm{s},(b)$ $170 \mathrm{~m} / \mathrm{s}$, and (c) $210 \mathrm{~m} / \mathrm{s}$. These micrographs are taken from the shear fracture surfaces of chips, as indicated in Fig. 5. 

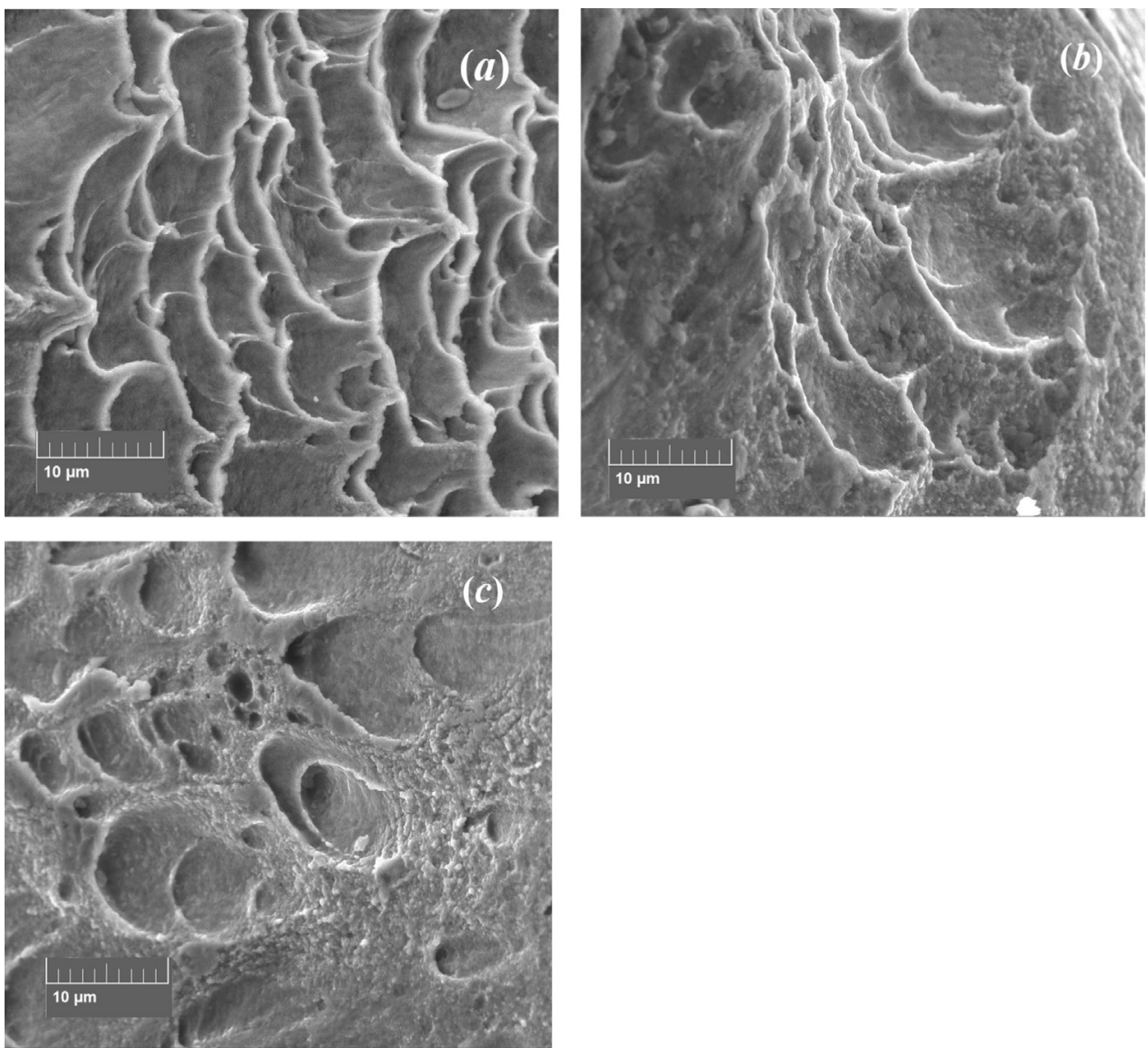

Fig. 7. SEM observations of the dimple structure in the shear-localized deformation region in the Ti6Al4V alloy HSM process. The cutting speeds are equal to (a) $90 \mathrm{~m} / \mathrm{s}$, (b) $170 \mathrm{~m} / \mathrm{s}$, and (c) $210 \mathrm{~m} / \mathrm{s}$. These micrographs are taken from the regions indicated in Fig. 6 .

structure developed in the separation moment of two adjacent sawteeth, as illustrated in Fig. 5. The high-magnification SEM micrographs (Fig. 7) show that the adiabatic shear deformation caused the material melting in the dimple zone.

The other zone has a flat surface with a larger area, which makes up the main portion of the shear fracture surface. With increased cutting speed, the area of flat surface zone increased, whereas that of the ductile dimple structure decreased. That is to say, the microscopic mechanisms of material failure on the shear fracture surface were successively changed with increased cutting speed. Fig. 8 shows highly magnified SEM micrographs of the microstructures of the zone. It visibly demonstrates the intergranular fracture that took place following the formation of adiabatic shear band on the surface. Therefore, the adiabatic shear banding and the subsequent shear intergranular fracture govern discontinuous chip formation in high-speed cutting of Ti6Al4V alloy.

Comparing the results in Fig. 8, we note that, when the cutting speed $V_{0}=90 \mathrm{~m} / \mathrm{s}$, no molten drops came into view on the grain surfaces (Fig. $8 a$ ); when $V_{0}=170 \mathrm{~m} / \mathrm{s}$, only a few micro-melting drops appeared on the grain surfaces (as shown by the ellipses in Fig. 8b); and when $V_{0}=210 \mathrm{~m} / \mathrm{s}$, the micro-melting drops were widely observed on the cracked grain surfaces (Fig. $8 c$ ). In these observations, the stretching of molten materials (as indicated by the arrows in these figures) characterizes the shear slip direction. Here, the micro-melting phenomenon on the grain surfaces deserves our attention. Although the underlying mechanism of the phenomenon has not been entirely understood, it is highly possible that both the significant plastic flow and the large shear strain rates produced by adiabatic shear bands promote the formation of micro-melting droplets on the grain surfaces. Perhaps a more credible explanation can be obtained from further high-speed cutting experiments of Ti6Al4V alloy.

\section{Theory}

\subsection{Theoretical model}

Since the chip morphology better represents the removal mechanism of chip materials, it is necessary to study the morphological transition and formation mechanisms of chips. The existent OCMs (Burns and Davies, 2002; Molinari et al., 2002; Ye et al., 2013) mainly concern the effect of shear localization on serrated chip formation and scarcely involve the two-dimensional effects, like heat conduction and convection, inertia, and applied loadings in cutting. Therefore, the purpose of this section is to develop a twodimensional continuum model for studying chip plastic flow and formation mechanisms. Fig. 9 illustrates the two-dimensional OCM 

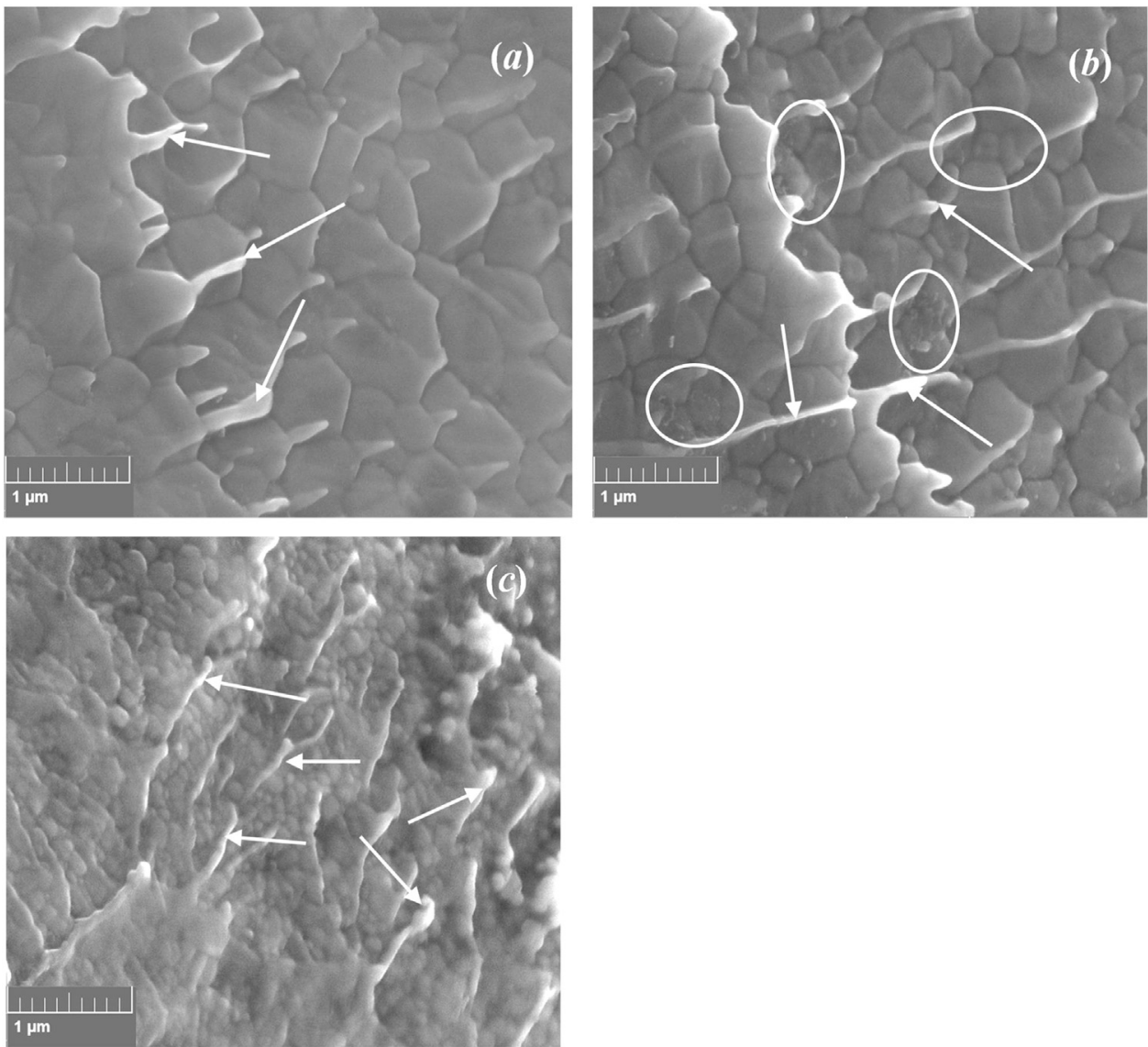

Fig. 8. SEM observations of the microstructures of smooth surface in the shear-localized deformation region in the Ti6Al4V alloy HSM process, where the cutting speeds are (a) $90 \mathrm{~m} / \mathrm{s},(b) 170 \mathrm{~m} / \mathrm{s}$, and (c) $210 \mathrm{~m} / \mathrm{s}$. These micrographs are taken from the regions indicated in Fig. 6.

following that of Ma et al. (2012). This model has an expanding chip formation zone $o A B C$ containing the PSZ and SSZ. The material in this zone is subjected to intensive transient stress loadings and large plastic deformation. Thus, it is appropriate to research the effect of the stress gradients from the tool rake face to the chip free surface and the frictional effect on the tool rake face. Most of our attention will be focused on the effects of normal stresses on the tool rake face (Burns and Davies, 1997) and the convection effect associated with chip flow (Gioia and Ortiz, 1996).

The cutting conditions are specified by rake angle $\alpha_{T}$, cut depth $h_{1}$, and cutting speed $V_{0}$. The shear plane on which the maximum shear strain arises is characterized by the shear angle $\varphi$, considered as a prescribed constant. The PSZ and SSZ have the thicknesses $\delta_{1}$ and $\delta_{2}$, respectively. Considering the largely deformed plastic flow and the convection motion of chip material in the HSM process, an analytical approach based on Eulerian formulation was used. As shown in Fig. 9, a Eulerian coordinate system attached to the tool

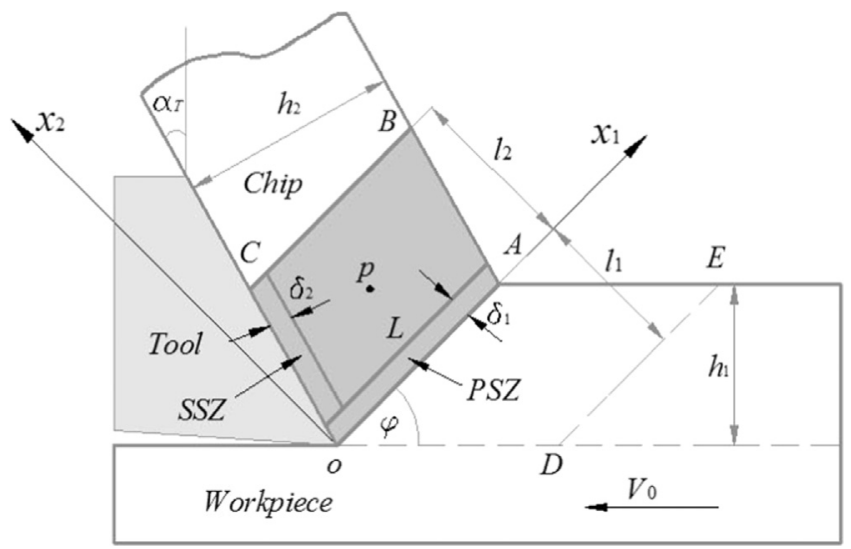

Fig. 9. The OCM with an expanding chip formation zone analyzed in the present study. 
tip was introduced. The $x_{1}$-axis was chosen in the direction of shear plane $o A$, and the $x_{2}$-axis is normal to it. Vectors $\mathbf{x}=\mathbf{x}\left(x_{i}\right)$ and $\mathbf{v}$ $=\mathbf{v}\left(x_{i}, t\right)$ denote the positions and the velocity field in the current configuration at time $t$, respectively.

In this model, the effects of heat conduction and convection on chip flow were considered. The cutting heat came from two sources: one was the large plastic deformation of work materials in the PSZ. We assumed that $90 \%$ of the plastic deformation work was converted into heat, inducing a temperature rise of the chip materials. The other source of cutting heat arose from friction between the tool and the chip. In the cutting of Ti6Al4V alloy, a small part of the heat was dissipated by heat conduction into the uncut workpiece material due to its low heat conductivity.

\subsection{The governing equations}

When studying the two-dimensional structure of shear bands in thermoviscoplastic solids, Gioia and Ortiz (1996) developed a general boundary layer theory that merely accounts for the two-dimensional effect of heat convection on shear band evolution. By defining a dimensionless parameter Re, named the generalized Reynolds number, the authors introduced a group of dimensionless variables and established Prandtl's boundary layer equations for characterizing the shear-localized instability. At this point, we followed their train of thought and analytical approach and introduced dimensionless variables

$$
\widehat{x}_{1}=\frac{x_{1}}{L}, \widehat{x}_{2}=\operatorname{Re} \frac{x_{2}}{L}, \hat{v}_{1}=\frac{v_{1}}{V_{0}}, \hat{v}_{2}=\operatorname{Re} \frac{v_{2}}{V_{0}}, \hat{t}=\frac{V_{0} t}{L}, \hat{\sigma}_{i j}=\frac{\operatorname{Re} \sigma_{i j}}{\rho V_{0}^{2}}, \widehat{T}=\frac{T-T_{0}}{T_{m}-T_{0}}
$$

to establish the mathematical formulation of the two-dimensional OCM (Fig. 9). Here $L, V_{0}, \rho, T_{0}$, and $T_{m}$ denote the PSZ length, the cutting speed, the mass density, the reference temperature, and the melting point of the work material, respectively. The dimensionless parameter Re, called the modified Reynolds number, is defined as

$$
\operatorname{Re}=\frac{\chi\left(V_{0}\right) \rho V_{0}^{2}}{\tau_{s}}
$$

where $\tau_{s}=A_{0} / \sqrt{ } 3$ is the shear yield stress of the work material. The parameter Re characterizes the inertia effect of workpiece motion. In the definition of parameter Re, we introduced the revised function $\chi\left(V_{0}\right)$ to modify the original parameter Re in Gioia and Ortiz (1996) for characterizing the two-dimensional flow behaviors of chip material. The function $\chi\left(V_{0}\right)$ is related to the cutting speeds and material properties, and thus it can be determined in terms of the cutting experiments and numerical tests. The experimental cutting results of the Ti6Al4V alloy in Fig. 3 show that the transition speed from the continuous chip to the serrated chip is about approximately $0.5 \mathrm{~m} / \mathrm{s}$. The numerical test of Molinari et al. (2013) shows that, when the cutting speed is approximately $350 \mathrm{~m} / \mathrm{s}$, the evolution of shear bands is restrained and the serrated chip changes back into a continuous chip. Therefore, in accordance with the sub/super-critical cutting speeds ( $V_{0 i}=0.5 \mathrm{~m} / \mathrm{s}$ and $V_{0 T}=350 \mathrm{~m} / \mathrm{s}$ ) at which the sawteeth in the chip appear and disappear, the function $\chi\left(V_{0}\right)$ is assumed to have the piecewise form of

$$
\chi\left(V_{0}\right)=\left\{\begin{array}{lr}
\frac{\tau_{s}}{\rho V_{0 i}^{2}}\left(\operatorname{Re}_{c}+\operatorname{Re}_{0} \frac{V_{0 i}^{2}-V_{0}^{2}}{V_{0}^{2}}\right) & \left(\operatorname{Re}_{0} \leq \operatorname{Re}<\operatorname{Re}_{c}\right) \\
\frac{\tau_{s}}{\rho} \frac{\operatorname{Re}_{c}}{V_{0}^{2}}-\left(1-\frac{V_{0 i}}{V_{0}}\right)\left(1-\frac{V_{0 T}}{V_{0}}\right) & \left(\operatorname{Re}^{2} \geq \operatorname{Re}_{c}\right)
\end{array},\right.
$$

where the critical parameter $\operatorname{Re}_{0}=1$ corresponds to the initial condition of $V_{0}=0$; the conditions $\operatorname{Re}_{c}=10$ and $V_{0 i}=0.5 \mathrm{~m} / \mathrm{s}$ denote the transition condition from continuous to serrated chips, and $\operatorname{Re}_{c}=10$ and $V_{0 T}=350 \mathrm{~m} / \mathrm{s}$ denote the transition condition from discontinuous to continuous chips. These conditions determine the serrated chip formation and disappearance, i.e., the initiation and the termination of adiabatic shear bands in the chip.

Now the parameter Re plays a role similar to the generalized Reynolds number used by Gioia and Ortiz (1996). By using the group of dimensionless variables in (1), we obtain the two-dimensional governing equations for chip flow as follows:

Momentum balance equation

$$
\begin{aligned}
& \dot{\hat{v}}_{1}+\widehat{v}_{1} \hat{v}_{1,1}+\hat{v}_{2} \hat{v}_{1,2}=\frac{1}{\operatorname{Re}} \widehat{\sigma}_{11,1}+\widehat{\sigma}_{12,2} \\
& \dot{\hat{v}}_{2}+\hat{v}_{1} \hat{v}_{2,1}+\hat{v}_{2} \hat{v}_{2,2}=\widehat{\sigma}_{12,1}+\operatorname{Re} \widehat{\sigma}_{22,2}
\end{aligned}
$$

Energy balance equation

$$
R_{v}\left(\hat{\dot{T}}+\widehat{v}_{1} \widehat{T}_{, 1}+\widehat{v}_{2} \widehat{T}_{, 2}\right)=R_{c}\left(\widehat{T}_{, 11}+\operatorname{Re}^{2} \widehat{T}_{, 22}\right)+R_{g} \hat{\dot{w}}
$$

Compatibility condition of deformation

$$
\widehat{\varepsilon}_{11,22}+\widehat{\varepsilon}_{22,11}=2 \widehat{\varepsilon}_{12,12}
$$

Continuity equation

$$
\widehat{v}_{1,1}+\widehat{v}_{2,2}=0
$$

In the energy eq. (5), $\hat{\dot{w}}=\widehat{\sigma}_{i j} \hat{\varepsilon}_{i j}(i, j=1,2)$ is dimensionless plastic power and the dimensionless parameters $R_{\boldsymbol{v}}, R_{\boldsymbol{c}}$, and $R_{\boldsymbol{g}}$ are defined in Eq. (10) below. In this paper, the hat “^” over a quantity denotes that it is dimensionless. The dot “.” over a quantity and 
the comma ", before subscript " $i$ " indicates the differentials in time and in coordinate $x_{i}$, respectively. In the above system of equations, the dimensionless strain and strain rate components are defined as follows:

$$
\begin{aligned}
& \hat{\varepsilon}_{11}=\frac{\partial \hat{u}_{1}}{\partial \widehat{x}_{1}}, \hat{\varepsilon}_{22}=\frac{\partial \hat{u}_{2}}{\partial \widehat{x}_{2}}, \hat{\varepsilon}_{12}=\frac{1}{2}\left(\operatorname{Re} \frac{\partial \hat{u}_{1}}{\partial \widehat{x}_{2}}+\frac{1}{\operatorname{Re}} \frac{\partial \hat{u}_{2}}{\partial \widehat{x}_{1}}\right) \\
& \hat{\dot{\varepsilon}}_{11}=\frac{\partial \hat{v}_{1}}{\partial \widehat{x}_{1}}, \hat{\dot{\varepsilon}}_{22}=\frac{\partial \hat{v}_{2}}{\partial \widehat{x}_{2}}, \hat{\varepsilon}_{12}=\frac{1}{2}\left(\operatorname{Re} \frac{\partial \hat{v}_{1}}{\partial \hat{x}_{2}}+\frac{1}{\operatorname{Re}} \frac{\partial \hat{v}_{2}}{\partial \widehat{x}_{1}}\right)
\end{aligned}
$$

According to the definition of the parameter Re given by Gioia and Ortiz (1996), when $\operatorname{Re} \geq 10$, the system of (4) -(7) is reduced to Prandtl's boundary layer equations:

$$
\begin{aligned}
& \dot{\hat{v}}_{1}+\widehat{v}_{1} \widehat{v}_{1,1}+\widehat{v}_{2} \widehat{v}_{1,2}=\frac{1}{\operatorname{Re}} \widehat{\sigma}_{11,1}+\widehat{\sigma}_{12,2} \\
& \hat{\sigma}_{22,2}=0 \\
& R_{v}\left(\dot{\hat{T}}+\widehat{v}_{1} \widehat{T}_{, 1}+\hat{v}_{2} \widehat{T}_{, 2}\right)=R_{c} \operatorname{Re}^{2} \widehat{T}_{, 22}+R_{g} \widehat{\sigma}_{12} \widehat{v}_{1,2} \\
& \hat{\dot{\varepsilon}}_{12}=\frac{\operatorname{Re}}{2} \widehat{v}_{1,2} \\
& \widehat{v}_{1,1}+\widehat{v}_{2,2}=0
\end{aligned}
$$

Equation system (4)-(7) describes the two-dimensional flow of chip material in the expanding chip formation zone $o A B C$ in the continuous chip case, whereas system (9) describes the shear localization process occurring in the PSZ.

In the normalization of Eqs. (4)-(9), we use the dimensionless parameter Re defined in Eq. (2) and introduce the following three dimensionless parameters:

$$
R_{v}=\frac{\operatorname{Re} c\left(T_{m}-T_{0}\right)}{V_{0}^{2}}, R_{c}=\frac{\operatorname{Re} \lambda\left(T_{m}-T_{0}\right)}{L \rho V_{0}^{3}}, R_{g}=\beta
$$

where $c$ and $\lambda$ denote the specific heat and coefficient of thermal conduction of the work material. $R_{v}$ denotes the heat convection effect produced by the chip plastic flow. $R_{c}$ denotes the heat conduction effect. The Taylor-Quinney coefficient $R_{g}$ denotes the heat conversion from the plastic work accomplished externally by the tool.

The relation between the revised Reynolds Re and the cutting speed $V_{0}$ is illustrated in Fig. 10 . When $\operatorname{Re} \geq \operatorname{Re}_{0}$ (i.e., $V_{0}>0$ ), the system of (4)-(7) characterizes the behavior of chip flow along the tool rake face in the plane strain state. When $\operatorname{Re} \geq \operatorname{Re}_{c}$ and $V_{0 i}<$ $V_{0}<V_{0 T}$, Re is defined as a quasi-velocity independent parameter. This means that the cutting speed does produce obvious influence on the chip formation process when continuous chips transform into serrated chips with increased cutting speed. However, the influence of the cutting speed is almost constant for the serrated chip formation, as the cutting speed changes within the high-speed cutting regime. In this case, Prandtl's boundary layer Eq. (9) characterize the evolution of adiabatic shear bands in the serrated chip, whereas the system of (4)-(7) describes the behaviors of chip flow along the tool rake face. In Section 6 below, we will use the system of Eq. (9) in combination with the Grady and Kipp (1987) model to investigate both the behavior of shear band instability in serrated chips and the chip formation mechanism from an energetic viewpoint. In Fig. 10, we also illustrate the variation curve of the generalized Reynolds number Re adopted by Gioia and Ortiz (1996). We can see that it is always a small quantity. Therefore, if it is directly adopted in the equation system of (4)-(7), the system is unsuitable for the description of HSM processes.

In order to compare the roles of the parameters $\mathrm{Re}, R_{v}$, and $R_{c}$, the relationships varying with the cutting speeds are illustrated in Fig. 11 for the Ti6Al4V alloy. It shows that the influence of thermal conduction $R_{c}$ is significant only in the low-speed cutting process when $V_{0}<5.5 \mathrm{~m} / \mathrm{s}$, and it is unrelated to the shear banding instability of chip materials. The heat convection $R_{v}$ always produces

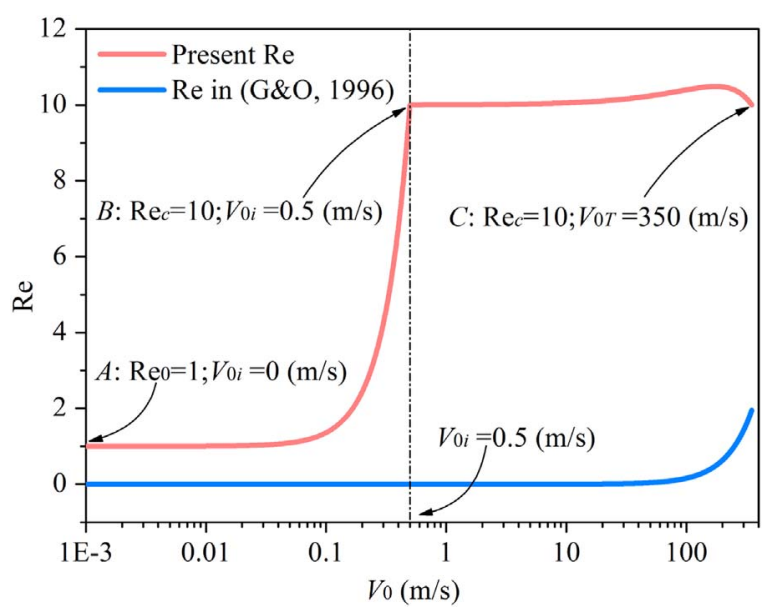

Fig. 10. The dependence of parameter Re on the cutting speed $V_{0}$. The Re used by Gioia and Ortiz (1996) is also shown with blue line for comparison. (For interpretation of the references to color in this figure legend, the reader is referred to the web version of this article.) 


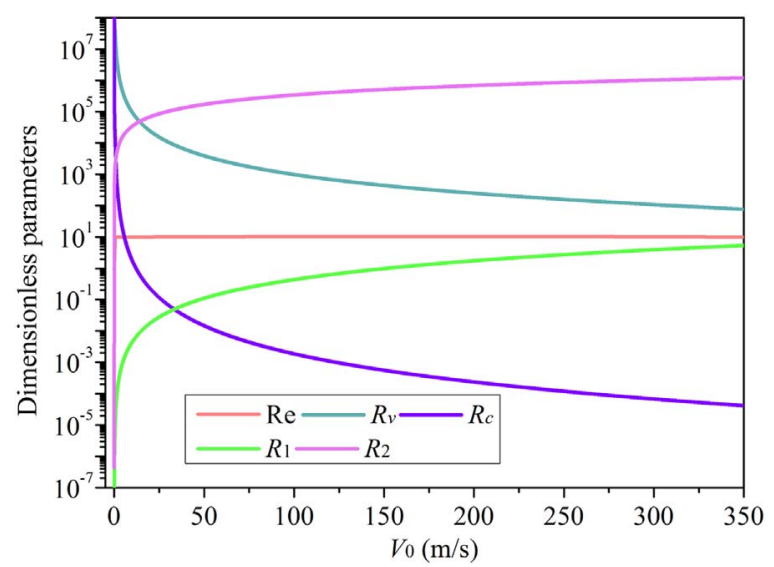

Fig. 11. Dependences of the dimensionless parameters $\operatorname{Re}, R_{v}, R_{c} R_{1}$, and $R_{2}$ on the cutting speed.

evident influence on the HSM process.

At present, the workpiece of Ti6Al4V alloy is assumed to obey a thermoviscoplastic law. The J-C constitutive model (Johnson and Cook, 1985) considers the effects of the strain hardening, rate sensitivity, and thermal softening of materials, and was thus adopted in the present analysis. The $\mathrm{J}-\mathrm{C}$ model in dimensionless form reads

$$
\widehat{\sigma}_{e q}=\left(\widehat{A}_{0}+\widehat{B}_{0} \hat{\varepsilon}_{e q}^{n}\right)\left(1+C_{0} \ln \frac{\hat{\dot{\varepsilon}}_{e q}}{\hat{\hat{\varepsilon}}_{0}}\right)\left(1-\widehat{T}^{m}\right),
$$

where $\widehat{\sigma}_{e q}, \hat{\varepsilon}_{\mathrm{eq}}, \hat{\varepsilon}_{\mathrm{eq}}$ and $\widehat{T}$ are the equivalent stress, equivalent strain, equivalent strain rate, and temperature in dimensionless form, respectively. $\widehat{\mathrm{A}}_{0}$ and $\widehat{\mathrm{B}}_{0}$ are the dimensionless material constants.

\section{Approximate flow fields in chip flows}

\subsection{Velocity fields}

When $V_{0}<V_{0 i}$, the chip flow is stationary; that is, the stress, velocity, and temperature inside the chip are time-independent. Thus, the field variables in the chip can be determined approximately with the expanding plastic zone model (Stevenson and Oxley, 1970-1971; Ma et al., 2012). Suppose that the uncut workpiece material in area $o D E A$ moves leftward into area $o A B C$ to form a chip segmentation (see Fig. 9). According to the results of Merchant (1945) and Shaw (1984), the relationships among cutting velocity $V_{0}$, chip velocity $V_{c}$, and shear velocity $V_{S}$ can be found from the velocity diagram of Fig. 12a and can be given in dimensionless form as follows:

$$
\widehat{V}_{C}=\frac{\sin \varphi}{\cos \left(\varphi-\alpha_{T}\right)}, \widehat{V}_{S}=\frac{\cos \alpha_{T}}{\cos \left(\varphi-\alpha_{T}\right)}, \widehat{V}_{N}=\sin \varphi
$$

The shear strain rate along the shear plane $o A$ and the compressive strain rate at the tool rake face $o C$ are given by Stevenson and
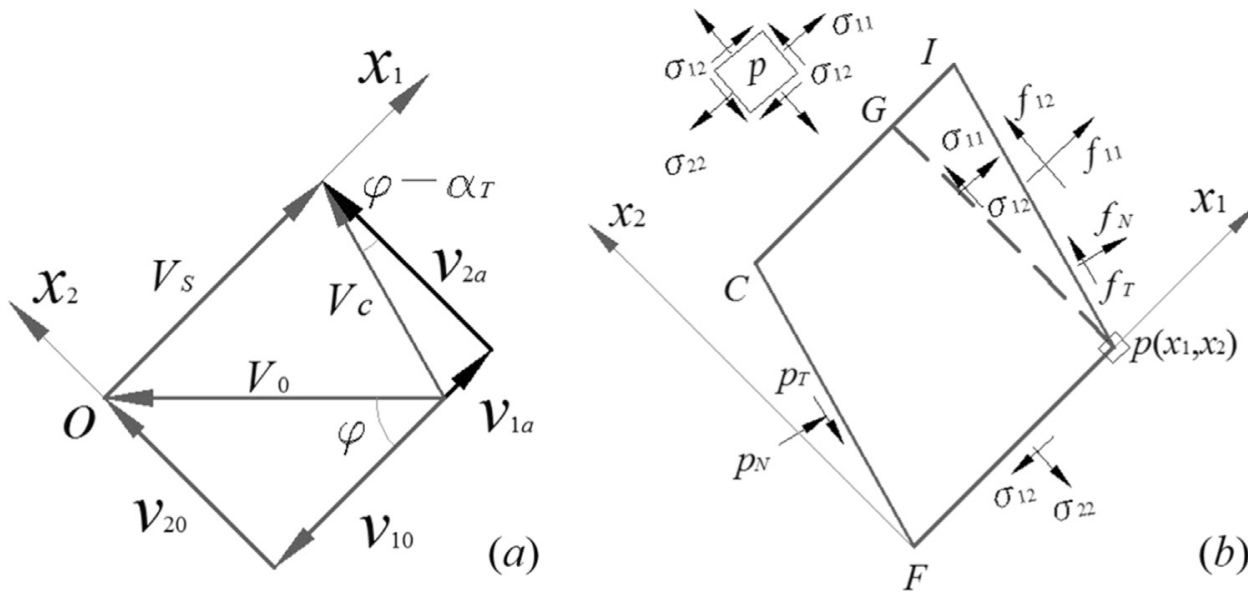

Fig. 12. (a) The relation between various velocity components; (b) the relation between stress components. 
Oxley (1970-1971) and Burns and Davies (1997), respectively; that is,

$$
\left.\widehat{\dot{\varepsilon}}_{12}\right|_{A B}=\frac{\cos \alpha_{T}}{\widehat{\delta}_{1} \cos \left(\varphi-\alpha_{T}\right)},\left.\hat{\dot{\varepsilon}}_{11}\right|_{o C}=\cos \varphi-\frac{\cos \alpha_{T}}{\cos \left(\varphi-\alpha_{T}\right)},
$$

where $\widehat{\delta}_{1}=0.17$ is the dimensionless width of PSZ given by Oxley and Welsh (1963). The boundary conditions satisfied by the velocity components are as follows:

$$
\left\{\begin{array}{lrl}
\widehat{x}_{2}=-\hat{l}_{1}: & \widehat{v}_{10}=-\cos \varphi, \widehat{v}_{20}=\sin \varphi \\
\widehat{x}_{2}=0: & \left.\widehat{v}_{1}\right|_{o A}=\frac{\cos \alpha_{T}}{\cos \left(\varphi-\alpha_{T}\right)},\left.\widehat{v}_{2}\right|_{o A}=\sin \varphi \\
\widehat{x}_{1}=\frac{1}{\operatorname{Re}} \tan \left(\varphi-\alpha_{T}\right) \widehat{x}_{2}: & \left.\widehat{v}_{1}\right|_{o C}=\sin \varphi \tan \left(\varphi-\alpha_{T}\right),\left.\widehat{v}_{2}\right|_{o C}=\sin \varphi \\
\widehat{x}_{2}=\hat{l}_{2}: & \widehat{v}_{1 a}=\sin \varphi \tan \left(\varphi-\alpha_{T}\right), \hat{v}_{2 a}=\sin \varphi
\end{array}\right.
$$

Roth (1969) showed that the stress and strain rates along the shear plane $o A$ reach peak values and that the stresses applied by the tool are uniformly distributed along the tool rake face $o C$. Furthermore, referring to compatibility conditions (6), (13), and (14), we obtain the velocity gradient components as follows:

$$
\begin{aligned}
& \left.\hat{v}_{1,2}\right|_{O A}=\frac{\cos \alpha_{T}}{\delta_{1} \operatorname{Re} \cos \left(\varphi-\alpha_{T}\right)},\left.\hat{v}_{2,1}\right|_{O A}=\frac{\operatorname{Re} \cos \alpha_{T}}{\delta_{1} \cos \left(\varphi-\alpha_{T}\right)} \\
& \left.\widehat{v}_{1,1}\right|_{O C}=-\left.\widehat{v}_{2,2}\right|_{o C}=\cos \varphi-\frac{\cos \alpha_{T}}{\cos \left(\varphi-\alpha_{T}\right)}
\end{aligned}
$$

To determine the velocity field in the chip formation area $O A B C$, we loosened the restrictive conditions on the velocity gradients in (15) along $O A$ and $o C$, that is, used them as boundary conditions, and integrated them to obtain the approximate velocity field as follows:

$$
\left\{\begin{array}{l}
\hat{v}_{1}=\widehat{v}_{1,1}\left(\widehat{x}_{1}-\frac{1}{2}\right)+\widehat{v}_{1,2}\left(\widehat{x}_{2}+\widehat{\delta}_{1} \operatorname{Re}\right) \\
\hat{v}_{2}=\hat{v}_{2,1}\left(\widehat{x}_{1}-\frac{\tan \left(\varphi-\alpha_{T}\right)}{2 \operatorname{Re}} \hat{l}_{2}\right)+\hat{v}_{2,2}\left(\widehat{x}_{2}-\frac{\hat{l}_{2}}{2}\right)+\frac{\sin \varphi}{\hat{l}_{2}}
\end{array}\right.
$$

where $\hat{l}_{2}=\sin \left(\varphi+\alpha_{\mathrm{f}}-\alpha_{\mathrm{T}}\right) / \cos \alpha_{\mathrm{f}}$ is the dimensionless tool-chip contact length (Oxley, 1989; see Fig. 9). The velocity field (16) approximately satisfies the boundary conditions on $o A$ and $o C$ in an average sense.

\subsection{Stress fields}

Following the works of Merchant (1945) and Burns and Davies (1997), the stress components $\sigma_{i j}$ of any point $p\left(x_{1}, x_{2}\right)$ in the chip formation zone (Fig. 9) can be approximately determined from the force-balance conditions of parallelogram $p I C F$ (Fig. 12b). Based on the assumption that the normal pressure $p_{N}$ is uniformly distributed along the tool rake face $o C$ (Roth, 1969) and Coulomb's law of friction, the tangential stress is defined as $p_{T}=p_{N} \tan \alpha_{f}$, where the friction angle $\alpha_{f}$ is constant. On the boundaries $A B$ and $B C$, the stress components are assumed to be free. Furthermore, in view of the formulation $p_{N}=-\rho c_{1} V_{0} / 2$ in one-dimensional stress wave theory, the dimensionless stress boundary conditions are given as follows:

$$
\begin{array}{lrl}
o C: \widehat{x}_{2}=\operatorname{Re} \widehat{x}_{1} \cot \left(\varphi-\alpha_{T}\right) ; \widehat{p}_{N}=-\frac{\operatorname{Re} \hat{c}_{1}}{2} \frac{\cos \alpha_{f}}{\cos \left(\varphi-\alpha_{T}\right)}, \widehat{p}_{T}=\frac{\operatorname{Re} \hat{c}_{1}}{2} \frac{\sin \alpha_{f}}{\cos \left(\varphi-\alpha_{T}\right)} \\
\left.\begin{array}{ll}
A B: \widehat{x}_{2}=\operatorname{Re}\left(\widehat{x}_{1}-1\right) \cot \left(\varphi-\alpha_{T}\right) \\
B C: \hat{x}_{2}=\hat{l}_{2}, D E: \hat{x}_{2}=-\hat{l}_{1}
\end{array}\right\}, & \hat{p}_{N}=\widehat{p}_{T}=0
\end{array}
$$

where $\hat{c}_{1}$ is the dimensionless speed of the longitudinal wave in a one-dimensional elastic bar. According to the Cauchy stress tetrahedron theorem, the traction components on the oblique plane $p I$ and the coordinate plane $p G$ (Fig. 12b) satisfy the relation of

$$
\left\{\begin{array}{l}
\widehat{f}_{N} \\
\widehat{f}_{T}
\end{array}\right\}=\left[\begin{array}{c}
-\hat{p}_{N} \\
\widehat{p}_{T}
\end{array}\right]+\widehat{L}_{o C}\left[\begin{array}{cc}
-\cos \left(\varphi-\alpha_{T}\right) & \sin \left(\varphi-\alpha_{T}\right) \\
\sin \left(\varphi-\alpha_{T}\right) & \cos \left(\varphi-\alpha_{T}\right)
\end{array}\right]\left\{\begin{array}{c}
\hat{\sigma}_{22} \\
\hat{\sigma}_{12}
\end{array}\right\} .
$$

From the boundary conditions on $o C$ and $A B$, the traction components on the plane $p I$ can be found as follows:

$$
\begin{aligned}
& \widehat{f}_{N}=-\frac{\widehat{p}_{N}}{\operatorname{Re}} \widehat{L}_{A B}, \widehat{f}_{T}=\frac{\widehat{p}_{T}}{\operatorname{Re}} \widehat{L}_{A B} \\
& \widehat{f}_{11}=-\widehat{f}_{N} \frac{\cos \left(\varphi+\alpha_{f}-\alpha_{T}\right)}{\cos \alpha_{f}}, \widehat{f}_{12}=-\widehat{f}_{T} \frac{\sin \left(\varphi+\alpha_{f}-\alpha_{T}\right)}{\sin \alpha_{f}}
\end{aligned}
$$

where

$$
\begin{aligned}
& \widehat{L}_{o C}=\sin \left(\varphi-\alpha_{T}\right) \frac{\operatorname{Re} \hat{x}_{1} \cot \left(\varphi-\alpha_{T}\right)-\hat{x}_{2}}{\hat{l}_{2}-\hat{x}_{2}} \\
& \widehat{L}_{A B}=\left[\operatorname{Re}\left(1-\widehat{x}_{1}\right)+\widehat{x}_{2} \tan \left(\varphi-\alpha_{T}\right)\right] .
\end{aligned}
$$

Furthermore, according to the quasi-static momentum equilibrium conditions, we obtain 


$$
\left\{\begin{array}{l}
\tan \left(\varphi-\alpha_{T}\right) \frac{\partial \hat{\sigma}_{12}}{\partial \hat{x}_{1}}+\operatorname{Re} \frac{\partial \hat{\sigma}_{12}}{\partial \hat{x}_{2}}=Y_{1} \\
\operatorname{Re} \tan \left(\varphi-\alpha_{T}\right) \frac{\partial \widehat{\sigma}_{12}}{\partial \widehat{x}_{2}}+\frac{\partial \hat{\sigma}_{12}}{\partial \widehat{x}_{1}}=Y_{2}
\end{array}\right.
$$

where parameters $Y_{1}$ and $Y_{2}$ are given in (A1) in Appendix A. Thus, once $\hat{\sigma}_{12}$ is found from Eq. (21), the normal stress components $\hat{\sigma}_{11}$ and $\hat{\sigma}_{22}$ in the chip can be found as follows:

$$
\left\{\begin{array}{l}
\widehat{\sigma}_{12}=\widehat{\Sigma}_{y}\left[I_{1}\left(1-\widehat{x}_{1}\right)+I_{2}\left(\hat{l}_{2}-\widehat{x}_{2}\right)\right] \\
\widehat{\sigma}_{11}=\widehat{\Sigma}_{y} \widehat{f}_{11} \cos ^{-1}\left(\varphi-\alpha_{T}\right)+\widehat{\sigma}_{12} \tan \left(\varphi-\alpha_{T}\right) \\
\widehat{\sigma}_{22}=\widehat{\Sigma}_{y} \widehat{f}_{12} \cos ^{-1}\left(\varphi-\alpha_{T}\right)+\widehat{\sigma}_{12} \tan \left(\varphi-\alpha_{T}\right)
\end{array}\right.
$$

where parameters $I_{1}$ and $I_{2}$ are also provided in (A1) in Appendix A. The stress components in (22) approximately satisfy the boundary conditions on $o C, A B$, and $B C$, and the shear stress component $\hat{\sigma}_{12}$ reaches its maximum value on shear plane $o A$. The constant $\widehat{\Sigma}_{\mathrm{y}}$ in (22) is determined by the condition that the shear stress at point $o$ equals the effective yield stress and satisfies the initial conditions $V_{0} \rightarrow 0$ or $\operatorname{Re}=\operatorname{Re}_{0}$ and $\hat{\sigma}_{\mathrm{ij}}=0$. Thus, we have

$$
\widehat{\Sigma}_{y}=\frac{\left(\operatorname{Re}-\operatorname{Re}_{0}\right)^{2}}{\operatorname{Re}} \frac{\widehat{A}_{0}+\widehat{B}_{0} \gamma_{c}^{n}}{I_{1}+I_{2} \hat{l}_{2}}
$$

where the critical strain $\gamma_{c}=0.6$ is taken from the analytic results of Molinari (1997).

From expressions of velocity and stress fields ((16) and (22)), we can see that the approximate fields are linear functions of the position coordinates $x_{1}$ and $x_{2}$. Therefore, we may believe that these results are the first-order approximation of the exact solution. These approximate formulations are valid for the description of chip plastic flows in the chip formation zone $O A B C$ (Fig. 9), though they are not directly applicable to the shear banding instability of materials in the PSZ.

\section{Linear stability analysis of chip flows}

Generally, the shear banding instability in serrated chip behaves as a strong localization of shear deformation and depends solely upon the shear stress and shear strain in the PSZ. On the contrary, the plastic instability of continuous chip flow depends upon the stress, strain, velocity, and temperature in an expanding chip formation zone. The difference between the two chip flow stabilities lies in the localization of the plastic deformation. In this section, we perform a linear stability analysis of chip flows in the twodimensional orthogonal cutting process, and obtain the corresponding instability criterion. In terms of the approximate flow fields derived in Section 4, the effects of applied stress loadings, rate sensitivity, and heat conduction and convection on the stability of chip flow are discussed, and the analytical results are further validated by numerical simulations.

\subsection{Instability criterion}

As stated above, if $\operatorname{Re}<10$, the governing equation system (4)-(7), together with the constitutive relation (11), describes the stability of continuous chip flows in plane strain state. If $\mathrm{Re}>10$, system (9) describes the shear banding instability in serrated chips. $\mathrm{Re}=10$ denotes the shape transition of continuous to serrated chips. Here, we make a linear stability analysis to gain a deeper understanding of the cutting process. We assume that systems (4)-(7) and the constitution Eq. (11) have the perturbation solution in the form of

$$
\left\{\widehat{\Psi}_{i j}\right\}=\left\{\widehat{\Psi}_{0 i j}\right\}+\left\{\delta \widehat{\Psi}_{i j}\right\} e^{\widehat{\omega} \hat{t}+i\left(\hat{k}_{1} \hat{x}_{1}+\hat{k}_{2} \hat{x}_{2}\right)}
$$

where

$$
\left\{\widehat{\Psi}_{i j}\right\}=\left\{\widehat{v}_{i}, \widehat{\sigma}_{i j}, \widehat{\varepsilon}_{i j}, \hat{\varepsilon}_{i j}, \widehat{T}\right\} \text { and }\left\{\widehat{\Psi}_{0 i j}\right\} \gg\left\{\delta \widehat{\Psi}_{i j}\right\} \text {. }
$$

Here $\widehat{\Psi}_{\mathrm{ij}}$ represents the inhomogeneous solution, $\widehat{\Psi}_{0 \mathrm{ij}}$ represents the homogeneous solution, and $\delta \widehat{\Psi}_{\mathrm{ij}}$ is the amplitude of perturbation on $\widehat{\Psi}_{0 \mathrm{ij}}$. The dimensionless wave vector is $\widehat{\mathbf{k}}_{0}=\hat{k}_{1} \mathbf{n}_{1}+\widehat{k}_{2} \mathbf{n}_{2}$, and the dimensionless wave number is $\hat{k}_{0}^{2}=\widehat{k}_{1}^{2}+\hat{k}_{2}^{2}$. In solutions (24) and (25), the perturbation of stress components $\delta \hat{\sigma}_{i j}$ can be found via differential operation of the constitution Eq. (11). Differentiating the constitutive relations and considering the cases of uniaxial tension and simple shear loadings, we obtain

$$
\delta \widehat{\sigma}_{i j}=\left\langle\widehat{Q}_{i j k l}\right\rangle\left\langle\delta \widehat{\varepsilon}_{k l}\right\rangle+\widehat{\omega}\left\langle\widehat{R}_{i j k l}\right\rangle\left\langle\delta \widehat{\varepsilon}_{k l}\right\rangle-\widehat{P}_{i j} \delta \widehat{T}
$$

where 


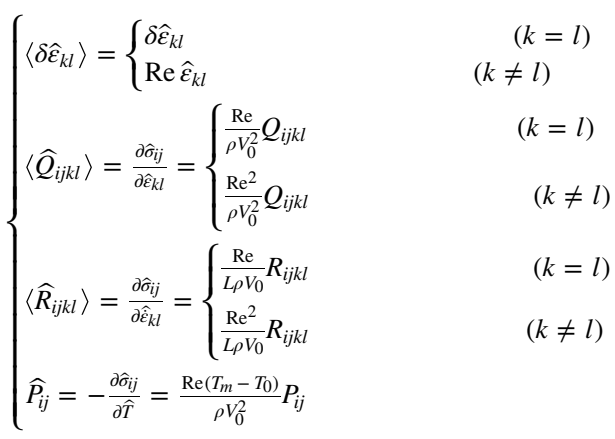

Here $\widehat{Q}_{\mathrm{ijk} l}, \widehat{\mathrm{R}}_{\mathrm{ijk} \mathrm{l}}$, and $\widehat{\mathrm{P}}_{\mathrm{ij}}$ denote the effects of strain hardening, rate sensitivity and thermal softening, respectively. Substituting the perturbation solution (24) and the micro-perturbed expression (26) of stress components into the system of Eqs. (4)-(7) and (11), and linearizing the resulting equations, we obtain a two-dimensional characteristic equation on the dimensionless perturbation growth rate $\widehat{\omega}$, as follows:

$$
J_{0} \widehat{\omega}^{3}+J_{1} \widehat{\omega}^{2}+J_{2} \widehat{\omega}+J_{3}=0
$$

The four coefficients $J_{0}, J_{1}, J_{2}$, and $J_{3}$ in (28) are given in (A2) of Appendix A. The dimensionless growth rate $\widehat{\omega}$ and wave number $\widehat{k}_{0}^{2}$ introduced in (24)-(28), and the two dimensionless parameters $R_{1}$ and $R_{2}$, are defined as follows:

$$
\widehat{\omega}=\frac{\lambda \omega}{c Q_{0}}, \hat{k}_{0}^{2}=\frac{\lambda^{2} k_{0}^{2}}{\rho c^{2} Q_{0}}, R_{1}=\frac{\rho V_{0}^{2}}{Q_{0}}, R_{2}=\frac{L \rho c V_{0}}{\lambda},
$$

where parameter $R_{1}$ is essentially the ratio of the inertial force to the strain hardening effect of solid materials, similar to the Reynolds number in fluid mechanics. Here, the strain hardening parameter $Q_{0}$ is defined in (A4) of Appendix A. It plays a role of resistance to the thermoplastic instability of materials. Parameter $R_{2}$, named the Reynolds thermal number by Bisacre et al. (1947), is essentially the ratio of the heat convected by a moving chip to the heat conducted to the workpiece and the tool. In Fig. 11, we also illustrate the dependences of $R_{1}$ and $R_{2}$ on the cutting speed. It can be seen that, in low-speed cutting, $R_{1}$ is a small quantity for the Ti6Al4V alloy due to the strong strain-hardening effect of the material. Only with cutting speed $V_{0}>10^{2} \mathrm{~m} / \mathrm{s}$ does the influence of $R_{1}$ become evident, implying that the thermal softening dominates the chip flow process. In contrast, $R_{2}$ is always a large quantity for the Ti6Al4V alloy due to its low heat conductivity. This suggests that the convection effect of a moving workpiece always has an appreciable influence on the cutting process. We can thus conclude that the machining of Ti6Al4V alloy is somewhat analogous to the adiabatic shear case, so serrated chips are easily developed in cutting.

The stability of chip flows depends on the value of growth rate $\widehat{\omega}$. If $\widehat{\omega} \leq 0$, the chip flow is stable; otherwise, plastic flow instability is possible. We can see that there are certainly some negative terms in the coefficient $J_{2}$ due to $C_{10}<0$ (see expression (A3) in Appendix A). These negative terms ensure that the characteristic Eq. (28) has at least one positive real root. This means that the chip plastic flow may develop continuously until plastic instability occurs at a special set of wave numbers (Bai, 1982). Therefore, based on the condition $d \widehat{\omega} / d \widehat{k}_{0}^{2}=0$, the criterion for evaluating the plastic instability of chip flow in plane strain state can be found as follows:

$$
\Xi=\frac{\left(F_{1}+F_{2} \widehat{R}_{0}+C_{Q} R_{2}^{-1} \widehat{\tau}_{c} F_{3}+2 R_{1} R_{2}^{-1} \widehat{\tau}_{c} F_{4}+R_{1} R_{c} R_{g}^{-1} \widehat{\tau}_{c} F_{5}\right) R_{g} \widehat{P}_{0}}{R_{v}\left(F_{6} \widehat{Q}_{0}+F_{7}\right)}>1,
$$

where a characteristic time $\hat{\tau}_{\mathrm{c}}$ is introduced. The seven functions $F_{i}(i=1, \ldots 7)$ in function $\Xi$ are given in expression (A5) of Appendix A. The dimensionless strain hardening $\widehat{Q}_{0}$, rate sensitivity $\widehat{R}_{0}$, and thermal softening $\widehat{P}_{0}$ are defined in expressions (A4) of Appendix A. In the criterion of (30), the numerator of function $\Xi$ represents the effect of thermal softening, and the denominator represents the effect of strain hardening and the contribution of heat convection to material hardening.

\subsection{Discussion}

First, consider the influences of various factors in the function $\Xi$ on thermal softening of chip materials. We can see that the numerator of the function involves the influence of five factors: applied stress $F_{1}$, rate sensitivity $F_{2} \widehat{R}_{0}$, heat conduction $C_{Q} R_{2}^{-1} \widehat{\tau}_{c} F_{3}$, heat convection $2 R_{1} R_{2}^{-1} \widehat{\tau}_{c} F_{4}$, and temperature gradients $R_{1} R_{c} R_{g}^{-1} \hat{\tau}_{c} F_{5}$. These factors govern the thermal softening behavior of chip materials. Fig. 13 illustrates the dependences of function $\Xi$ on Re. Curve (1) merely takes into account the contribution of applied stress $F_{1} R_{g} \widehat{P}_{0}$ to the thermal softening, and curve (2) further considers the influences of all five factors. The two curves overlap atRe $=2.26$. When $\operatorname{Re} \geq 2.26$, the rate sensitivity and heat convection lead to appreciable material hardening, resulting in a decrease in thermal softening. When $\operatorname{Re}<2.26$, the influence of these factors on thermal softening is not significant.

The stability of chip flow is easy to assess in two cases. When $\operatorname{Re}_{0}<\operatorname{Re}<1.14$ and $\Xi<1$, the continuous chip flow is stable. When $\operatorname{Re}>\operatorname{Re}_{c}$ and $\Xi>1$, shear banding instability takes place in the PSZ, and serrated chips form in an unstable cutting process. However, in the intermediate case of $1.14<\mathrm{Re} \leq \mathrm{Re}_{c}$, the condition $\Xi>1$ is still held. This suggests that the flow of continuous chips is possibly unstable. To ensure the stability of continuous chip flow in this case, we further consider the variations of chip 


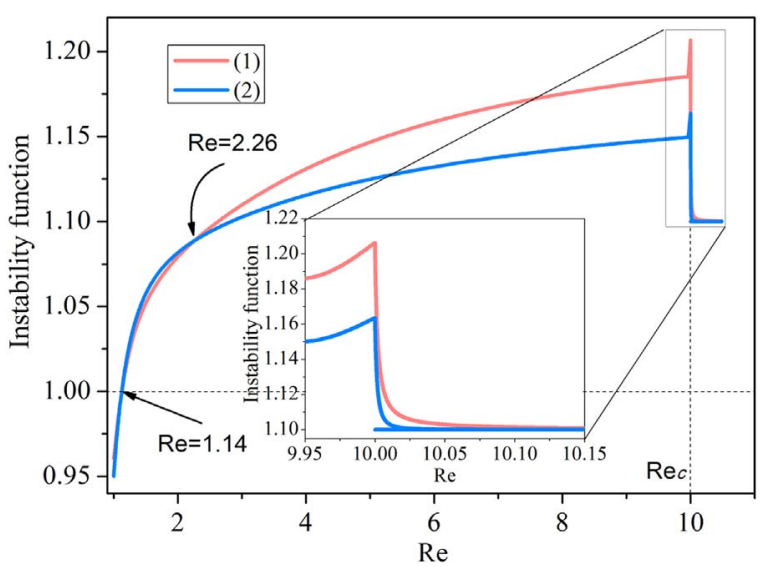

Fig. 13. The relationship between the instability function $\Xi$ and the Reynolds number Re for different influencing factors.
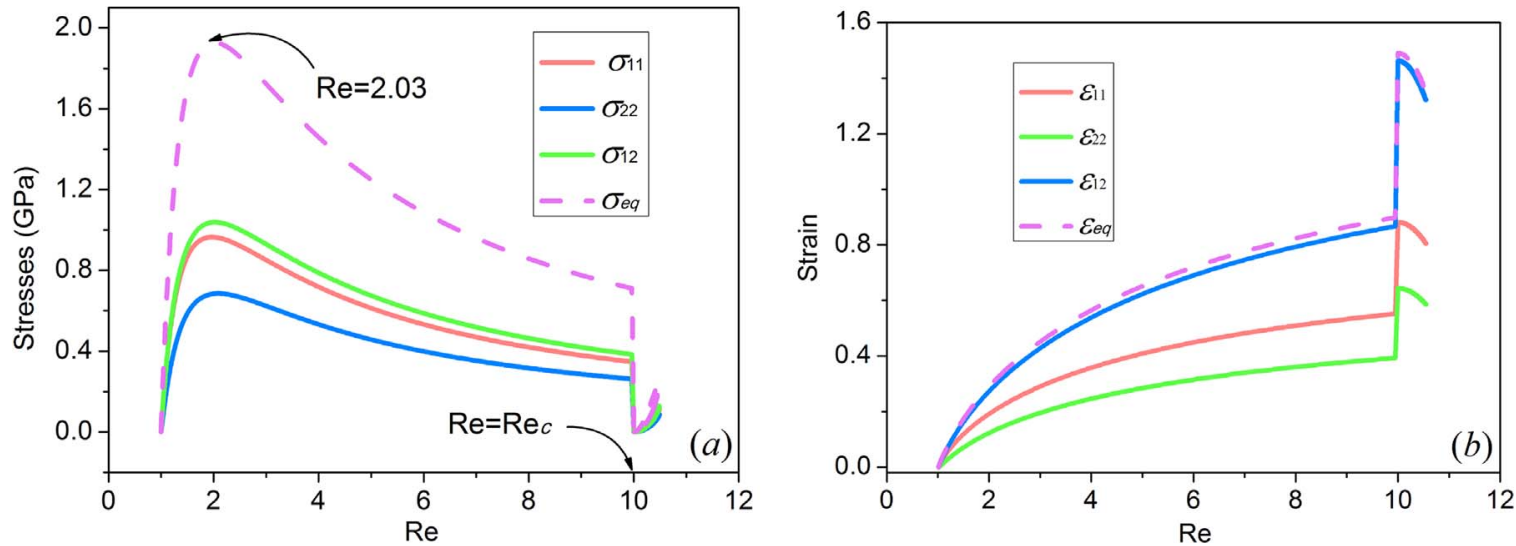

Fig. 14. The variations of stresses $(a)$ and strains $(b)$ with Re.

stresses and strains with parameter Re in the chip formation zone.

Figs. $14 a$ and $14 b$ demonstrate, respectively, the dependences of equivalent stress and equivalent strain of the chip on the parameter Re. When Re increases, the equivalent stress increases and arrives at its peak value ofRe $=2.03$. Then, it starts to decrease gradually until the shear banding instability takes place at $R e=\operatorname{Re}_{c}$, at which point the stress attains the minimum (Fig. 14a). In the meantime, the equivalent strain monotonically increases with Re until the maximum strain is reached at the moment when the shear banding instability occurs (Fig. 14b). Fig. 15 illustrates the relationship between the equivalent stress and equivalent strain. We can see that when $\mathrm{Re}>2.03$, the stress in the chip decreases with increasing strain, implying that thermal softening dominates the plastic flow of chip material. Therefore, we can deduce that the continuous chip flow is unstable when $2.03<\operatorname{Re}<\operatorname{Re}_{c}$. The strain

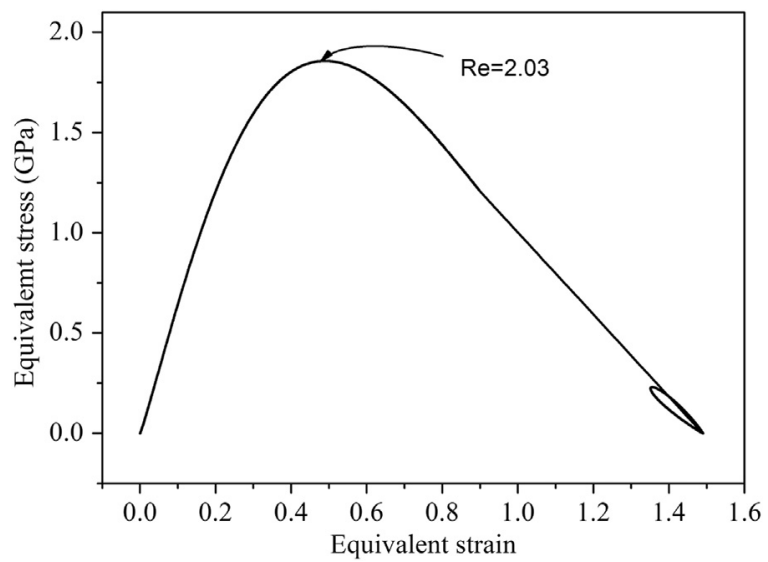

Fig. 15. The relation between the equivalent stress and equivalent strain of chip material in the chip formation zone. 

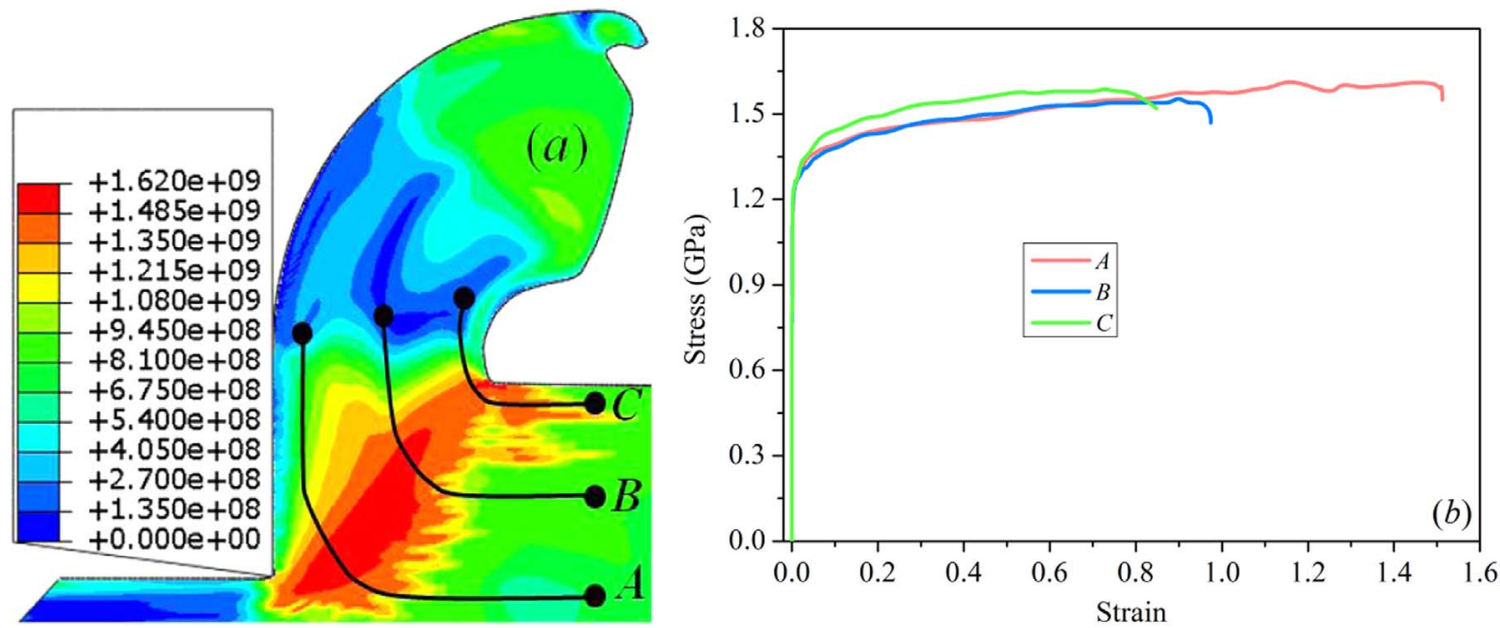

Fig. 16. (a) Contour of the equivalent stress of the continuous chip in the stable cutting process; $(b)$ the equivalent stress-strain curves of three material points $A, B$, and $C$ inside the chip. $V_{0}=0.05 \mathrm{~m} / \mathrm{s}$.

corresponding to instability is approximately 0.48 , and the critical instability speed is $V_{0 c}=0.175 \mathrm{~m} / \mathrm{s}$.

However, when $1.14<\operatorname{Re}<2.03$, the stress-strain relation in Fig. 15 demonstrates that the plastic flow of chip material is in the regime of strain hardening. This is an inconsistency with the material softening behavior predicted by the value of function $\Xi$ being larger than 1 (Fig. 13). Hence, the stability of continuous chip flow cannot be determined at this moment. One reason is that the flow field described in Section 4 is a first-order approximation, not an exact solution of the problem. Furthermore, we can see that the stable flow of continuous chip changes into an unstable one in the range of $1.14<\mathrm{Re}<2.03$. In the actual cutting process, the transition of chip morphology itself is a continuous event occurring over a short interval of time. Therefore, the intermediate range of $1.14<\operatorname{Re}<2.03$ denotes approximately the transition process. We have seen that, in two-dimensional orthogonal cutting, the plastic instability of continuous chip flows depends on the field variables of the plane strain state. Once the cutting speed arrives at a critical speed (e.g., $V_{0 c}=0.175 \mathrm{~m} / \mathrm{s}$ for the Ti6AL4V alloy), the continuous chip flow will lose its stability.

To further clarify the stability of continuous chip flows, numerical simulations of the orthogonal cutting of Ti6Al4V alloy have been performed using the Lagrangian finite element method. The details can be found in Ma et al. (2012). Two cutting cases are modeled:

Case (i): $V_{0}=0.05 \mathrm{~m} / \mathrm{s}$ andRe $=1.09$. In such a slow cutting process, the continuous chip flow is stationary, and all of the field variables in the chip and the workpiece are time-independent. Fig. $16 a$ illustrates the contour of the equivalent stress, which shows that the high amplitude and heterogeneous stresses arise in a larger plastic deformation zone surrounding the PSZ. Fig. 16b shows the equivalent stress-strain curves of three material points, $A, B$, and $C$, inside the chip (see Fig. 16a). It shows that the chip plastic flow exhibits hardening behavior as the material points of chip move in the formation zone and the stresses in chip do not approach zero ultimately. Hence, the continuous chip flow is stable.

Case (ii): $V_{0}=0.2 \mathrm{~m} / \mathrm{s}$ and $\mathrm{Re}=2.44$. With the increase in cutting speed, the heat conduction effect becomes slighter, and the
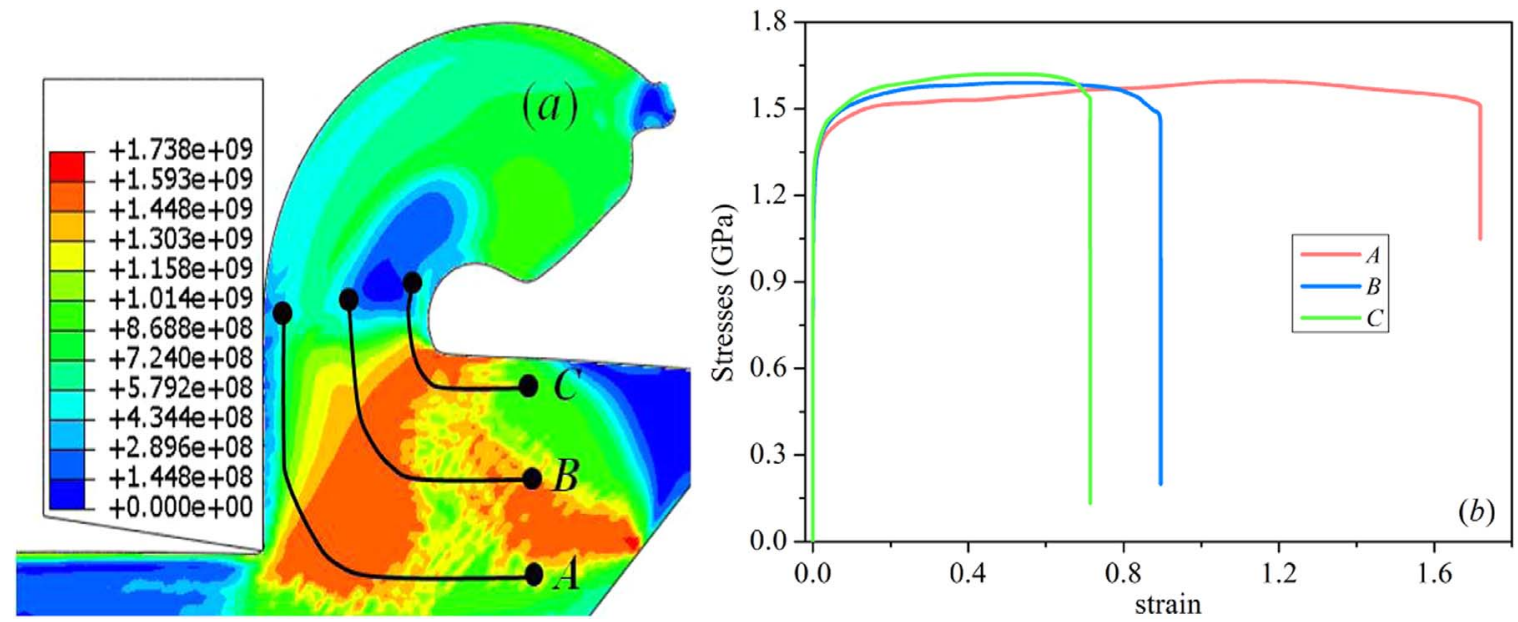

Fig. 17. (a) Contour of the equivalent stress of the continuous chip in the unstable cutting process; $(b)$ the equivalent stress-strain curves of three material points $A$, $B$, and $C$ inside the chip. $V_{0}=0.2 \mathrm{~m} / \mathrm{s}$. 


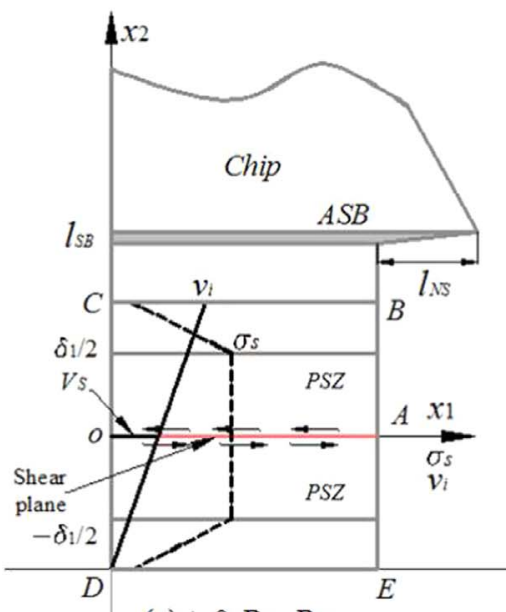

(a) $t=0 ; \mathrm{Re}^{-}=\mathrm{Re}_{c}-$

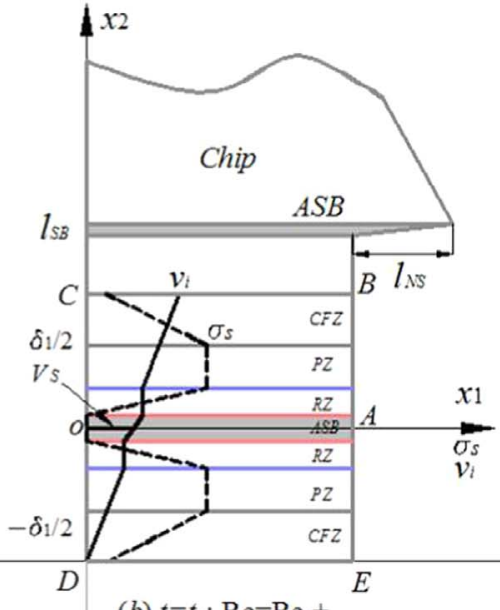

(b) $t=t c ; \operatorname{Re}=\operatorname{Re}_{c}+$

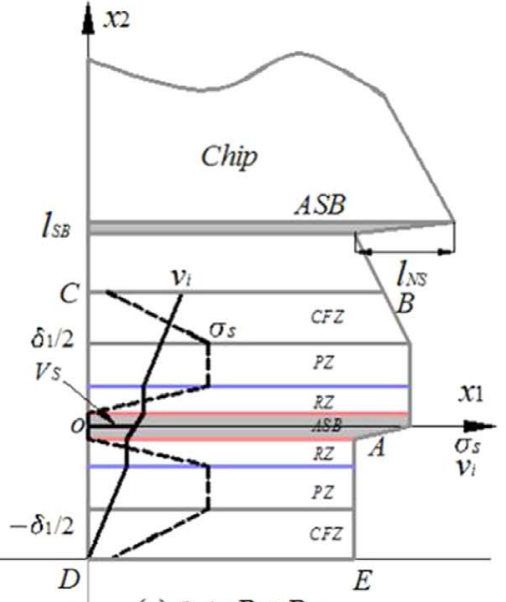

(c) $t>t c ; \operatorname{Re}>\operatorname{Rec}$

Fig. 18. The dynamic balance-based chip formation model corresponding to the OCM with an expanding chip formation zone. Figures (a) and (b) correspond to the initial and the terminal moments of the adiabatic shear band evolution, respectively, and figure (c) to the serrated chip evolution process.

adiabatic effect of plastic flow becomes more evident. In this situation, the effect of thermal softening controls the stability of chip flow. The equivalent stress contour in Fig. 17a shows that the equivalent stress field of the chip formation zone is heterogeneous. The stress-strain curves at $A, B$, and $C$ (Fig. 17b) show that the effective stresses first reach their peak values and then experience a plastic flow with rapid stress relaxation due to material softening, which leads to the loss of bearing capacity of chip material. Therefore, the continuous chip flow is unstable, and at this moment, the localization process is slow and has no time to develop. We have seen that the modeling cutting speed $V_{0 c}=0.2 \mathrm{~m} / \mathrm{s}$ is greater than the theoretical speed $V_{0 c}=0.175 \mathrm{~m} / \mathrm{s}$. This is because the numerical calculation models the case where the plastic instability has already occurred, while the theoretical prediction estimates merely the initiation of plastic instability. Whether or not, we can still come to the conclusion that, in the HSM process, there must be a critical speed above which the continuous chip flow becomes unstable.

\section{Formation of the discontinuous chips}

In Section 4, we obtained the approximate flow fields of chip plastic deformation. The flow fields can better characterize the twodimensional effect of chip flow, but be incapable of solving the issues involving strain localization processes, nor describe the evolution from the incipience of instability to full localization or serrated and discontinuous chips formation. Therefore, we need to follow another train of thought to consider these issues. This section begins with a description of various modes of energy consumption and relevant energy criteria during orthogonal cutting. Then, the Prandtl's boundary layer Eq. (9) in combination with the dynamic model of Grady and Kipp (1987) are used to investigate chip formation mechanisms from an energetic viewpoint.

\subsection{Energy criteria of chip formation}

For the continuous, serrated, and discontinuous chips, the mechanisms of energy dissipation are different. Fig. 18 shows the processes of shear-localized deformation and serrated chip formation. When the continuous chip forms $\left(\operatorname{Re}_{0}<\operatorname{Re}<\operatorname{Re}_{c}\right)(\mathrm{Fig}$. $18 a)$, the work done by cutting force $W^{T}$ is consumed in four ways: (i) the plastic deformation energy of chip $E^{P}$, (ii) the energy dissipated by friction between the tool and chip $E^{F}$, (iii) the kinetic energy of chip $E^{K}$, and (iv) the machined surface energy $E^{M S}$. From the nucleation to fully matured evolution of shear bands $\left(\mathrm{Re}=\mathrm{Re}_{c}\right)$, the sawteeth do not form on the chip (Fig. 18b). Taking into account the heat conversion of plastic work of material inside the shear bands, the energy dissipated by the chip should also include (v) the shear-localized deformation energy $E^{S B}$. During the development of the serrated chip $\left(\operatorname{Re}>\operatorname{Re}_{c}\right.$ ), the new surface denotes that the sawteeth has formed on the free surface of the chip (Fig. 18c). Then, the energy dissipated by the serrated chip should also be included: (vi) the new surface energy $E^{N S}$. Thus, the global energy balance in serrated chip formation can be expressed as follows:

$$
\widehat{W}^{T}=\widehat{E}^{P}+\widehat{E}^{F}+\widehat{E}^{K}+\widehat{E}^{M S}+\widehat{E}^{S B}+\widehat{E}^{N S}
$$

where the relation (31) has been nondimensionalized by the quantity $\operatorname{Re} / \rho L^{2} V_{0}^{2}$. In Table 2, we list the range of the parameter Re, energy balance conditions or energy criteria, and the chip shape in the cutting process.

In Table 2, $\widehat{E}^{S B}=0^{+}$means that the shear band starts to nucleate, $\widehat{E}_{m}^{S B}$ is the saturated limit of adiabatic shear deformation energy $\widehat{E}^{S B}, \widehat{\gamma}_{S}=\gamma_{S} \operatorname{Re} / \rho L^{2} V_{0}^{2}$ is the dimensionless surface energy per unit area, and $\hat{l}_{N S}$ is the dimensionless length of the new surface (see Fig. $18 b$ and $c$ ). Referring to the occurrence of shear band instability, the cutting process can be divided into three stages: no shear bands arising for $\operatorname{Re}_{0}<\operatorname{Re}<\operatorname{Re}_{c}$, shear band nucleation to sufficiently matured evolution of shear bands for $\mathrm{Re}_{c}^{-} \leq \mathrm{Re}^{\mathrm{s}} \leq \mathrm{Re}_{c}^{+}$, and occurrence of shear fracture for $\operatorname{Re}>\operatorname{Re}_{c}$. The corresponding energy criteria and conditions are given by energy relationships [1] to 
Table 2

Summary of the parameter Re range, energy balance conditions or energy criteria, and the chip shape.

\begin{tabular}{|c|c|c|c|}
\hline Parameter Re & Energy balance conditions ${ }^{\mathrm{a}}$ & Chip shape & Annotation \\
\hline $\operatorname{Re}_{0}<\operatorname{Re}<\operatorname{Re}_{c}$ & $\widehat{E}^{S B}=0, \widehat{E}^{N S}=0$ & Continuous & No adiabatic shear band develops in the chip. \\
\hline $\operatorname{Re}=\operatorname{Re}_{c}^{-}$ & $\widehat{E}^{S B}=0^{+}, \widehat{E}^{N S}=0[1]$ & Continuous & Nucleation of adiabatic shear band starts in the PSZ. \\
\hline $\operatorname{Re}_{c}^{-}<\operatorname{Re}<\operatorname{Re}_{c}^{+}$ & $0<\widehat{E}^{S B}<\widehat{E}_{m}^{S B}, \widehat{E}^{N S}=0[2]$ & Continuous & Nucleation of adiabatic shear band is in progress. \\
\hline $\operatorname{Re}=\operatorname{Re}_{c}^{+}$ & $\begin{array}{l}\widehat{E}^{S B}=\widehat{E}_{m}^{S B} \\
\widehat{E}^{N S}=\widehat{E}_{2}^{K}=0^{+}\end{array}$ & Continuous & Nucleation of adiabatic shear band has just terminated, and serrated chip starts to develop. \\
\hline $\operatorname{Re}>\operatorname{Re}_{c}$ & $\begin{array}{l}\widehat{E}^{S B}=\widehat{E}_{m}^{S B}, \\
0<\widehat{E}^{N S}=\widehat{E}_{2}^{K}=\hat{\gamma}_{S} \hat{l}_{N S}, \quad \text { 4] } \\
\hat{l}_{N S}<1\end{array}$ & Serrated & $\begin{array}{l}\text { Development of serrated chip is in progress, and new surface forms on the chip-free } \\
\text { surface. }\end{array}$ \\
\hline $\operatorname{Re}>\operatorname{Re}_{c}$ & $\begin{array}{l}\widehat{E}^{S B}=\widehat{E}_{m}^{S B} \\
\widehat{E}^{N S}=\hat{\gamma}_{S}, \quad[5] \\
\hat{l}_{N S}=1\end{array}$ & Discontinuous & The development of serrated chip terminates, and the discontinuous chip starts to form. \\
\hline
\end{tabular}

[1] The energy criterion for the onset of shear bands.

[2] The energy condition for the evolution of shear bands.

[3] The energy criterion for sawtooth initiation in the serrated chip.

[4] The energy condition for sawtooth growth in the serrated chip.

[5] The energy criterion for the initiation of discontinuous chips.

${ }^{a}$ When the condition $\widehat{E}^{s b}=\widehat{E}^{P}, \widehat{E}^{N S}=0$ is substituted into (31), it changes into the energy balance condition for the continuous chip.

[5] listed in Table 2. In the serration growth process, the kinetic energy of a chip segmentation is divided into two parts: $\widehat{E}^{K}=\widehat{E}_{1}^{K}+\widehat{E}_{2}^{K}$, in which $\widehat{\mathrm{E}}_{1}^{\mathrm{K}}$ denotes the residual kinetic energy of chip segmentation moving with the speed of $\widehat{\mathrm{V}}_{\mathrm{c}}$ and $\widehat{\mathrm{E}}_{2}^{\mathrm{K}}$ is the kinetic energy to be converted into the new surface energy $\widehat{\mathrm{E}}^{\mathrm{NS}}$.

According to the theoretical results of Shaw (1984) and Atkins (2003), the work accomplished externally by the cutting force is given as follows

$$
\widehat{W}^{T}=\frac{\hat{\tau}_{b} \hat{l}_{S B} \cos \left(\alpha_{f}-\alpha_{T}\right)}{\sin ^{2} \varphi \cos \left(\varphi+\alpha_{f}-\alpha_{T}\right)\left[1-\tan \left(\varphi+\alpha_{f}-\alpha_{T}\right)\right]},
$$

where $\hat{1}_{\mathrm{SB}}$ is the dimensionless shear band spacing, and $\tau_{b}$ is the shear strength of the workpiece material. The plastic deformation energy of the chip is as follows:

$$
\widehat{E}^{P}=\int_{0}^{\operatorname{Re} \hat{l}_{S B}} \frac{d \widehat{x}_{2}}{\operatorname{Re}} \int_{\widehat{o C}}^{\widehat{A B}} \hat{\sigma}_{i j} \widehat{\varepsilon}_{i j} d \widehat{x}_{1},
$$

where the dimensionless stress components $\hat{\sigma}_{\mathrm{ij}}$ are given in (22), and the dimensionless strain components $\hat{\varepsilon}_{\mathrm{ij}}$ can be solved from the constitution relationship (11) without considering the thermal softening effect. The friction energy on the tool rake face is given as follows:

$$
E^{F}=\frac{\hat{\tau}_{b} \hat{l}_{S B} \sin ^{2} \varphi}{\cos \left(\varphi-\alpha_{T}\right) \cos \left(\varphi+\alpha_{f}-\alpha_{T}\right)\left[1-\tan \left(\varphi+\alpha_{f}-\alpha_{T}\right)\right]} .
$$

When the chip is fully developed, the residual kinetic energy in the chip segmentation is

$$
\widehat{E}^{K}=\widehat{E}_{1}^{K}=\frac{\operatorname{Re} V_{c}^{2} \hat{l}_{S B}}{2} \text {. }
$$

In the serration growth process, the kinetic energy that is converted into the new surface energy $\widehat{\mathrm{E}}^{\mathrm{NS}}$ is given as follows:

$$
\widehat{E}_{2}^{K}=\frac{\operatorname{Re} \hat{l}_{S B}}{2}\left[\overline{\hat{v}}_{1}^{2}-\overline{\hat{v}}_{1 c}^{2}-\widehat{V}_{c}^{2} \sin ^{2}\left(\varphi-\alpha_{T}\right)\right],
$$

where average velocity $\overline{\mathrm{v}}_{1}$ is obtained from (16) by integrating, and $\overline{\mathrm{v}}_{1 \mathrm{c}}$ is the critical velocity at $\operatorname{Re}=\mathrm{Re}_{\mathrm{c}}^{+}$. The surface energy due to the formation of machining surfaces is

$$
\widehat{E}^{S}=\widehat{\gamma}_{S} \hat{l}_{S B}\left(\frac{1}{\sin \varphi}+\frac{1}{\cos \left(\varphi-\alpha_{T}\right)}\right) .
$$

For ductile metal cutting, the partition of cutting energy has been estimated by Shaw (1984). The shear deformation energy of chip material is approximately $73 \%$ of the total cutting energy, while approximately $27 \%$ is consumed in friction, and less than $1 \%$ is transformed to the machining surface and the kinetic energy of the chip. The former two parts of energy are estimated to be of the order of $10^{-2} \mathrm{~J} / \mathrm{m}^{2}$ in view of (33) and (34), and the latter two parts are approximately of the order of $10^{-5} \mathrm{~J} / \mathrm{m}^{2}$, referring to (35) to 
(37). Therefore, the latter two parts of energy are usually negligible in steady cutting analyses. However, in the dynamic analysis of high-speed cutting, the two components of energy, i.e., the machining surface energy and the kinetic energy, can still be used to describe the sawtooth growth behavior in terms of the energy criteria [2] to [4] listed in Table 2 (Atkins, 2003). For this purpose, it is necessary to first perform the analysis of the shear-localized instability of chip flow to determine the shear band spacing $\hat{l}_{S B}$, shear band width $\widehat{\delta}_{S B}$, shear-localized deformation energy $\widehat{E}^{S B}$, and saturated limit $\widehat{E}_{m}^{S B}$. This will be performed using the dynamic model of Grady and Kipp (1987).

\subsection{Analysis of shear-localized deformation}

In Fig. 18, we illustrate the formation process of a serrated chip due to the shear-localized deformation of material in the PSZ. A Lagrangian coordinate system $\left(x_{1}, x_{2}\right)$ is attached to the tool tip. The $x_{1}$ coordinate axis is parallel to the shear plane, and the $x_{2}$ axis is perpendicular to it. Consider the portion of material above the shear plane in the half space $x_{2} \geq 0$, and assume that the velocities and displacements of material points are constantly zero on the bottom boundary $D E$. Before the shear-localized deformation takes place, the chip is continuous. The velocity of material point in the chip formation zone $V_{1}$ is assumed to be along $x_{1}$ direction and changes linearly from zero on the lower boundary to the chip velocity $V_{S}$ on the shear plane, as shown in Fig. $18 a$. The shear stress is homogeneous throughout the PSZ. Since the shear band spacing, shear band width, and PSZ width are all small quantities, the field variables in these zones are identified as linearly dependent on coordinate $x_{2}$. During the evolution of shear bands, momentum diffusion leads to the extension of a rigid zone. For $t>0$, the position of the rigid-plastic boundary is denoted by the Lagrangian coordinate $x_{2}=\zeta(t)$ (Fig. $18 b$ and $c$ ). Then, the dimensionless velocity component in the $x_{1}$ direction is given as follows:

$$
\widehat{V}_{1}=\left\{\begin{array}{lc}
\widehat{V}_{S}+2 \hat{\dot{\varepsilon}}_{12} \widehat{x}_{2} & 0 \leq \widehat{x}_{2}<\frac{1}{2} \operatorname{Re} \widehat{\delta}_{S B} \\
\widehat{V}_{S}+\hat{\dot{\varepsilon}}_{12} \widehat{\zeta} & \frac{1}{2} \operatorname{Re} \widehat{\delta}_{S B} \leq \widehat{x}_{2}<\operatorname{Re} \widehat{\zeta} \\
2 \widehat{V}_{S}+2 \hat{\dot{\varepsilon}}_{12} \widehat{x}_{2} & \operatorname{Re} \hat{\zeta} \leq \hat{x}_{2}<\operatorname{Re} \hat{l}_{S B}
\end{array}\right.
$$

Based on this velocity field and according to the analytical approach of Grady and Kipp (1987), we may find the characteristic time of shear localization process as follows:

$$
\hat{t}_{c}=\left[\frac{9 \operatorname{Re} \widehat{E}^{S B 2}}{2 \hat{\varepsilon}_{12} \hat{\tau}_{s}^{3}}\left(1+\sqrt{1+\left(\frac{\hat{\tau}_{s} \widehat{V}_{S}}{3 \widehat{E}^{S B}}\right)^{2}}\right)\right]^{\frac{1}{3}}
$$

The critical shear band spacing is

$$
\hat{l}_{S B c}=2 \widehat{\zeta}=\frac{\hat{\tau}_{s}^{2}}{3 \operatorname{Re} \widehat{E}_{m}^{S B}} \hat{t}_{c}^{2}+\widehat{\delta}_{S B c}-\frac{\widehat{V}_{S}}{2 \hat{\dot{\varepsilon}}_{12}} .
$$

Based on the energy balance Eq. (9), we obtain the shear-localized deformation energy as follows:

$$
\widehat{E}^{S B}=\frac{R_{v} \widehat{\delta}_{S B c}}{R_{g}}\left(1+\frac{\overline{\hat{v}}_{2} \hat{t}_{c}}{\widehat{\delta}_{S B C}}+\frac{R_{c} \operatorname{Re}^{2} \hat{t}_{c}}{2 \widehat{\delta}_{S B C}^{2}}\right)
$$

Furthermore, from the requirement of maximum softening rate, we may determine the minimum localized time as follows:

$$
\hat{t}_{c m}=\frac{2 R_{v} \widehat{\delta}_{S B c}^{2}}{R_{c}} .
$$

The corresponding saturated limit of shear-localized deformation energy is found to be

$$
\widehat{E}_{m}^{S B}=\frac{R_{v} \widehat{\delta}_{S B c}}{R_{g}}\left(1+R_{v} \mathrm{Re}^{2}\right) .
$$

Finally, the critical shear band width can be given as

$$
\widehat{\delta}_{S B c}=\left(\frac{9 \operatorname{Re}_{c} R_{c}^{3}}{2 R_{v} R_{g}^{2} \hat{\varepsilon}_{12} \hat{\tau}_{s}^{3}}\right)^{\frac{1}{4}} .
$$

Now we have obtained all the desired results, i.e., from Eq. (39) to Eq. (44). For the sake of brevity, the specific derivation procedure of these results is described in Appendix B.

\subsection{Results and discussion}

Eq. (44) demonstrates that the critical shear band width $\hat{\delta}_{\mathrm{SBc}}$ is related to the strain rate, cutting conditions, and work-material properties. Fig. 19a shows the dependence of the critical width $\hat{\delta}_{\mathrm{SBc}}$ on the critical Reynolds number $\mathrm{Re}_{c}$. We can see that the effect of parameter $\operatorname{Re}_{c}$ on width $\widehat{\delta}_{S B c}$ is very weak. The approximate relation between the critical width $\hat{\delta}_{\mathrm{SBc}}$ and the cutting speed can be 

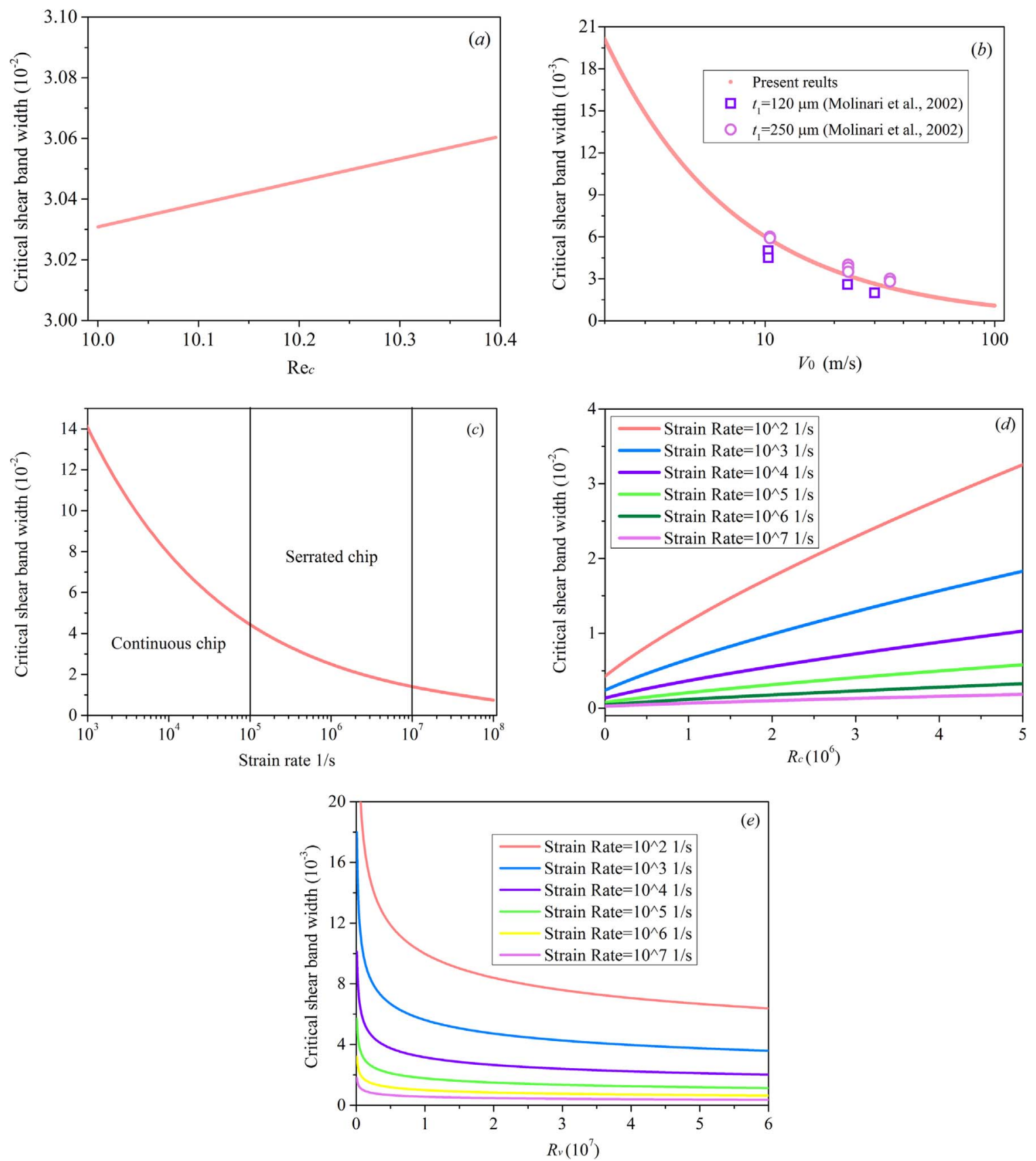

Fig. 19. The critical shear band width versus the critical Reynolds number $\operatorname{Re}_{c}(a)$, the critical velocity $V_{0 c}(b)$, the shear strain rate $(c)$, and the dimensionless thermal parameters $R_{c}(d)$ and $R_{v}(e)$. In Fig $(b), t_{1}=120 \mu \mathrm{m}$ and $t_{1}=250 \mu \mathrm{m}$ are the cut thickness.

found from (44) to be $\widehat{\delta}_{\mathrm{SBc}} \propto 1 / \mathrm{V}_{0}^{3 / 4}$. This result is close to $\widehat{\delta}_{S B c} \propto 1 / V_{0}$, which was used by Wright and Ockendon (1996) and Molinari et al. (2002) for estimating the shear band width. Fig. $19 b$ similarly shows the weak dependences of the critical width $\hat{\delta}_{\mathrm{SBc}}$ on the cutting velocity $V_{0}$. In this figure, the results of Molinari et al. (2002) are shown with discrete points. We can see that their results are in good agreement with the present prediction. In the cutting of Ti6Al4V alloy, the strain rate of the shear-localized deformation is usually in the range of $10^{5} 1 / \mathrm{s}$ to $10^{7} 1 / \mathrm{s}$. Fig. $19 \mathrm{c}$ shows that the critical width $\hat{\delta}_{\mathrm{SBc}}$ decreases as the strain rate $\dot{\varepsilon}_{12}$ increases, but it is not very sensitive to the strain rate. In Figs. $19 \mathrm{~d}$ and e, we illustrate the influences of the dimensionless parameters $R_{c}$ and $R_{v}$ on the critical width. When $\dot{\varepsilon}_{12}>10^{5} 1 / \mathrm{s}$, the influences of $R_{c}$ and $R_{v}$ on the critical width are negligible. However, when $\dot{\varepsilon}_{12}<10^{3} 1 / \mathrm{s}$, the critical width quickly increases as $R_{c}$ increases. This implies that heat conduction is one of the crucial factors that influence the chip flow process in low cutting-speed processes.

The experimental results of Meyers et al. (2001) and Xue et al. (2002, 2004) demonstrate that both the perturbation theory 

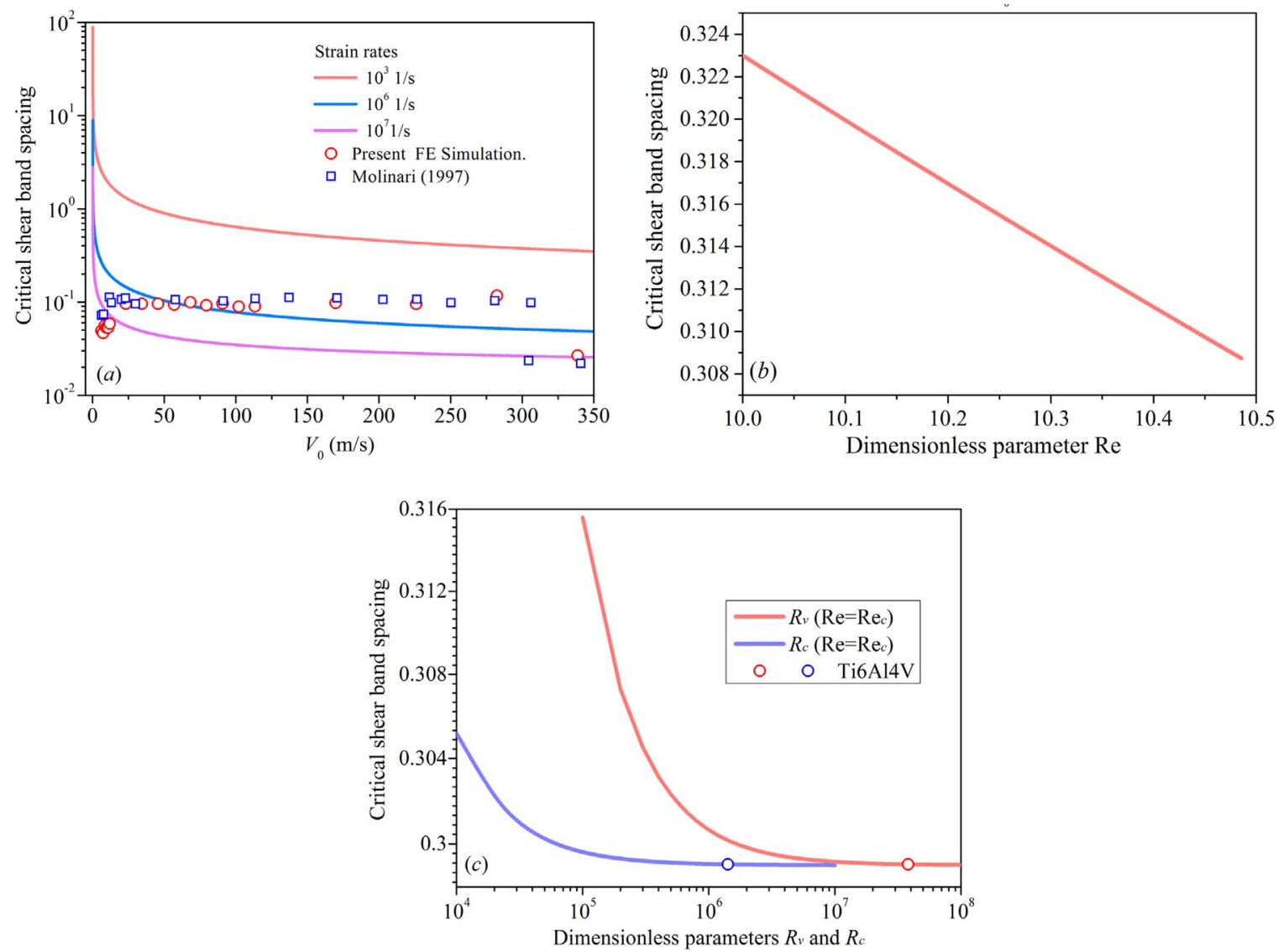

Fig. 20. The critical shear band spacing versus the cutting speed $V_{0}$ for different shear strain rates $(a)$, versus parameters Re $(b)$ and versus parameters $R_{v}$ and $R_{c}(c)$.

(Molinari, 1997; Wright and Ockendon, 1996) and the momentum diffusion theory (Grady and Kipp, 1987) can better predict the shear band spacing $\hat{l}_{S B C}$. Eq. (40) predicts that the average spacing of shear bands in the chips of Ti6Al4V alloy is approximately $50 \mu \mathrm{m}$, which is consistent with the experimental observations in Figs. 2 and 3. The results in Fig. 20a show that the critical spacing decreases with increasing cutting speed. Here, the perturbation analysis results (blue squares) of Molinari (1997) and our numerical calculation results (red circles) are also provided for comparison. We can see that the theoretical predictions of shear band spacing approach the numerical results in the range of cutting speeds from $20 \mathrm{~m} / \mathrm{s}$ to $120 \mathrm{~m} / \mathrm{s}$. The deviation arises when the cutting speed is above $120 \mathrm{~m} / \mathrm{s}$. In the HSM process with the cutting speed below $300 \mathrm{~m} / \mathrm{s}$, both Molinari's and our numerical results show a constant critical spacing that approaches the present prediction with the strain rate level of $10^{6} 1 / \mathrm{s}$. When the cutting speed is above $300 \mathrm{~m} / \mathrm{s}$, the critical spacing decreases and approaches the prediction with the strain rate of $10^{7} 1 / \mathrm{s}$. These results are coincident with the experimental observations shown in Fig. 3. Fig. $20 b$ demonstrates that the variation of critical spacing with Re is negligible (less than 5\%). Similarly, the variations with $R_{v}$ and $R_{c}$ (Fig. 20c) show that the heat-transfer effects by convection and conduction on the critical spacing are not significant when cutting Ti6Al4V alloy. These results are consistent with the predictions of Show (1987) and Atkins (2003).

The dimensionless length $\hat{l}_{N S}$ of new surface characterizes the growth of the sawteeth in serrated chips. Fig. 21a shows the relationship between the length and the cutting speed obtained from the kinetic energy Eq. (36) and the energy criteria [3] and [4] listed in Table 2. Fig. $21 b-f$ provide the contours of equivalent plastic strains of various shape chips produced at different cutting speeds. On this curve, the conditions $V_{0}=V_{0 i}$ andRe $=\operatorname{Re}_{c}$ at point $A$ have multiple implications: the conditions $V_{0}<V_{0 i}$ and $\operatorname{Re}<\operatorname{Re}_{\mathrm{c}}^{-}$denote continuous chip formation (Fig. 21b); $V_{0}=V_{0 i}$ and $\mathrm{Re}=\mathrm{Re}_{c}^{-}$denote the nucleation of a shear band at the tool tip (Fig. 21c); and $V_{0}=V_{0 i}$ and $\mathrm{Re}=\mathrm{Re}_{c}^{+}$denote the fully matured evolution of a shear band and the initiation of sawtooth growth in the chip (Fig. 21d). At the moment, the new surface length on the chip-free surface satisfies the condition $\hat{l}_{N S}=0^{+}$. On segment $A B$ of the curve, the conditions $V_{0 i}<V_{0}<75 \mathrm{~m} / \mathrm{s}$ and $\operatorname{Re}_{c}<\mathrm{Re}<10.2$ characterize the growth of sawteeth in serrated chips, and thus the condition $0<\hat{l}_{N S}<1$ is held (Fig. 21e). On segments $B C$, the conditions $V_{0}>75 \mathrm{~m} / \mathrm{s}$ and Re $>10.2$ imply that the discontinuous chip has developed and thus $\hat{l}_{N S}>1$ (Fig. 21f). Point $B$ on the curve $\hat{l}_{N S}=1$ denotes the transition from serrated chips to discontinuous chips and points $B^{+}, C$, and $D$ denote that discontinuous chips have developed. The predicted transition speed of $75 \mathrm{~m} / \mathrm{s}$ falls in the region of the experimental measurement shown in Figs. 2 and 3.

In order to describe the localization degree of shear deformation in the serrated chips, Molinari et al. (2013) introduced a geometrical parameter, called segmentation index $S i$, to characterize the irregular chip segment shape. The definition of $S i$ is based 

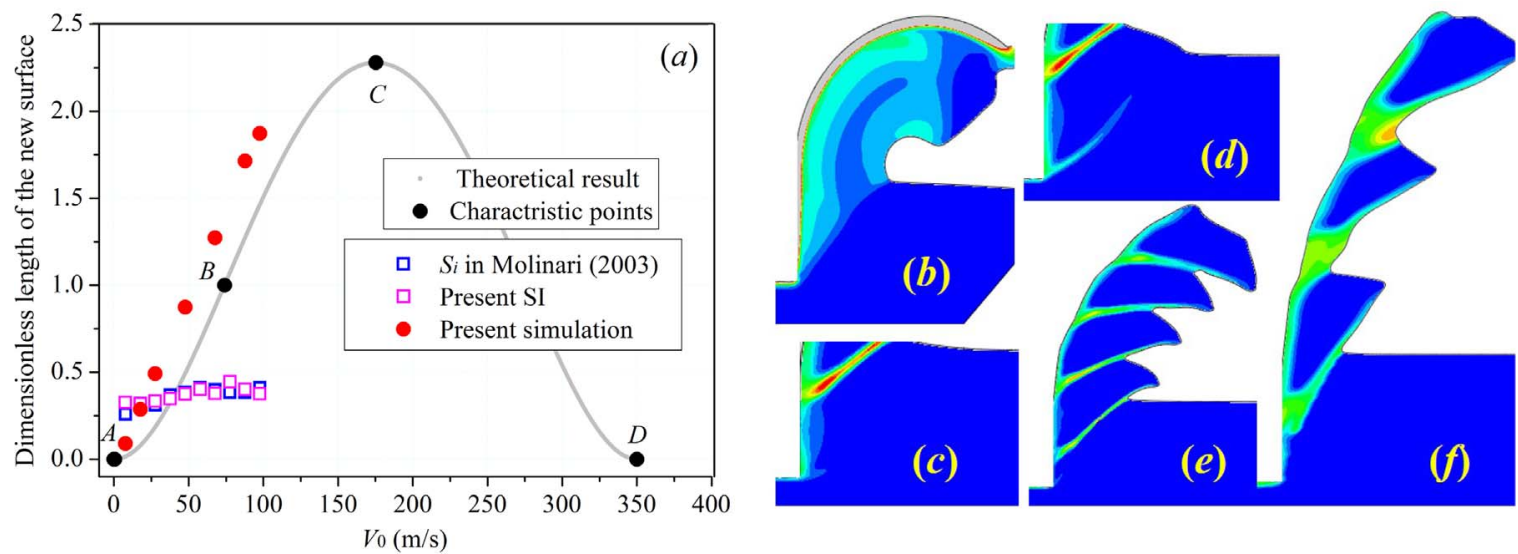

Fig. 21. The theoretical and numerical results of the new surface length versus the cutting speed $V_{0}(a)$. Contours of equivalent strain implying the formation of continuous chips $(b)$, the nucleation of adiabatic shear band at tool tip $(c)$, the growth of sawteeth in serrated chips $(d)$, the development of serrated chips $(e)$, and the development of discontinuous chips $(f)$.

on the average distances from the peaks and valleys of chip free surface to the tool-chip contact surface. In Fig. $21 a$, the results of Molinari et al. (2013) and the present calculations in terms of the segmentation index $\mathrm{Si}_{\text {i }}$ as well as the present simulation results using the finite element method, are shown with discrete points. We can see that the former roughly describe the mean size of the sawteeth, and the latter have better consistency with the theoretical prediction of serrated and discontinuous chips.

As stated above, part of the kinetic energy of chip segmentation $\widehat{E}_{2}^{K}$ is converted into the new surface energy of the discontinuous chip. From the energy criterion [4] in Table 2, we can see that the new surface energy $\widehat{\mathrm{E}}^{\mathrm{NS}}$ arrives at the maximum $\widehat{E}_{m}^{N S}=\widehat{\gamma}_{S}$ and $\hat{1}_{\mathrm{NS}}=1$ at the moment when the discontinuous chip begins to form. When the kinetic energy $\widehat{E}_{2}^{\mathrm{K}}$ exceeds the maximum surface energy $\widehat{\mathrm{E}}_{\mathrm{m}}^{\mathrm{NS}}$, the discontinuous chip can not only fully develop but also fly off from the tool surface in the direction of the shear plane due to the residual kinetic energy $\widehat{E}_{2}^{K}-\widehat{E}_{m}^{N S}$ remaining in the chip segmentation. This situation can be clearly observed by comparing the modeling results shown in Fig. $21 e$ and $f$.

\section{Conclusion}

Cutting experiments were carried out by using a Ti6Al4V alloy with cutting speeds ranging from 0.05 to $210 \mathrm{~m} / \mathrm{s}$. The microscopic observations of chips reveal the transition of morphology from continuous to serrated and then to discontinuous chips. It has shown that the different mechanisms, i.e., plastic instability, shear deformation localization, and subsequent shear intergranular fracture of chip material, result in chip formation with different shapes.

Based on the experimental observations, an improved orthogonal cutting model in the plane strain state was proposed to take into account the two-dimensional effects of chip flows in metal cutting. Through a dimensional analysis, the major dimensional parameters controlling the stability of chip flow were obtained. Theoretical analysis and numerical simulation reveal that, for the Ti6Al4V alloy, the heat conduction effect is significant only in the low-speed cutting process, while the influence of heat convection on the HSM process is always evident. Different from the case of shear band instability in PSZ, the plastic instability of continuous chip flows along the tool rake face occurred in an expanding chip formation zone, and no evident shear deformation localization developed. The corresponding instability criterion was derived via perturbation analysis and then used to evaluate the plastic instability of chip flows and determine the corresponding critical speed for the transition from stable to unstable chip flows.

The energy dissipation of the chip material in the HSM process was analyzed for the evolution of continuous, serrated, and discontinuous chips. Based on energy balance between the kinetic energy and the surface energy of the chip, the corresponding energy criteria were proposed and used to characterize the sawtooth growth behavior and the formation mechanism of discontinuous chips.

This study shows that the two-dimensional orthogonal cutting model put forward here expands the application range of traditional one-dimensional models in a few aspects, including the descriptions of chip flow and formation, the heat convection effect of chip flow, and the pressure stress influence on tool rake faces. However, this model still has sufficient room for further improvement. For example, for better study of the physical essence in metal cutting, an original dimensionless parameter (or several) should be used to establish new models. The parameter should not be artificially modified as defined in (2). Furthermore, it is necessary to consider the nonlinear effects of approximate flow fields of material in the chip formation zone and the influence of plastic deformation of work material on chip formation mechanisms.

\section{Acknowledgments}

The authors are grateful for the financial support from the Key National Nature Science Foundation of China (Grant no. 
11132011) and the National Nature Science Foundation of China (Grant nos. 11572337 and 51575029).

\section{Appendix A}

In this appendix, the expressions of the constants and functions presented in the main context are provided.

The coefficients in the expressions (21)-(23) are as follows:

$$
\begin{aligned}
& Y_{1}=\frac{1}{I_{0}} \operatorname{Re} \cos \varphi\left[\tan ^{2}\left(\varphi-\alpha_{T}\right)-1\right] \\
& Y_{2}=\frac{1}{I_{0}} \operatorname{Re} \sin \varphi \tan \left(\varphi-\alpha_{T}\right)\left[\tan ^{2}\left(\varphi-\alpha_{T}\right)-1\right] \\
& I_{1}=\frac{1}{I_{0}} \operatorname{Re} \tan \left(\varphi-\alpha_{T}\right)(\cos \varphi-\sin \varphi) \\
& I_{2}=\frac{1}{I_{0}}\left[\cos \varphi-\sin \varphi \tan ^{2}\left(\varphi-\alpha_{T}\right)\right] \\
& I_{0}=2 \cos \left(\varphi-\alpha_{T}\right) \cos \left(\alpha_{f}-\alpha_{T}\right)\left[1-\tan ^{2}\left(\varphi-\alpha_{T}\right)\right]
\end{aligned}
$$

\section{Appendix B}

In this appendix, we present the details of the analysis process and derivation procedure of the results presented in Eqs. (39) to (44) in subsection 6.2.

In terms of the analysis of the plastic flow behavior of material in the chip formation zone at the beginning of the subsection, we established a velocity field as follows:

$$
\widehat{V}_{1}=\left\{\begin{array}{lc}
\widehat{V}_{S}+2 \hat{\dot{\varepsilon}}_{12} \widehat{x}_{2} & 0 \leq \widehat{x}_{2}<\frac{1}{2} \operatorname{Re} \widehat{\delta}_{S B} \\
\widehat{V}_{S}+\hat{\dot{\varepsilon}}_{12} \widehat{\zeta} & \frac{1}{2} \operatorname{Re} \widehat{\delta}_{S B} \leq \widehat{x}_{2}<\operatorname{Re} \widehat{\zeta} \text { where the average velocity } \overline{\widehat{v}}_{1}\left(x_{2}\right)=\widehat{V}_{S}+2 \hat{\dot{\varepsilon}}_{12} \widehat{x}_{2} \text { has been used. } \\
2 \widehat{V}_{S}+2 \hat{\dot{\varepsilon}}_{12} \widehat{x}_{2} & \operatorname{Re} \hat{\zeta} \leq \widehat{x}_{2}<\operatorname{Re} \hat{l}_{S B}
\end{array}\right.
$$

The dimensionless momentum of material per unit area of shear band is as follows:

$$
\widehat{P}_{m}=\left(\widehat{V}_{S}+\frac{1}{2} \operatorname{Re} \hat{\dot{\varepsilon}}_{12} \widehat{\delta}_{S B}\right) \operatorname{Re} \widehat{\delta}_{S B}+\widehat{\zeta}\left(\widehat{V}_{S}+2 \hat{\dot{\varepsilon}}_{12} \widehat{\zeta}\right)+\left(\hat{l}_{S B}-\widehat{\zeta}\right)\left[2 \widehat{V}_{S}+\hat{\dot{\varepsilon}}_{12}\left(\hat{l}_{S B}+\widehat{\zeta}\right)\right],
$$

where $\widehat{P}_{m}=P_{m} / \rho V_{0} L$ is the dimensionless momentum. The time rate of the momentum is

$$
\widehat{\hat{P}}_{m}=\left(\widehat{V}_{S}+\hat{\dot{\varepsilon}}_{12} \operatorname{Re} \widehat{\delta}_{S B}\right) \operatorname{Re} \hat{\dot{\delta}}_{S B}+\left(2 \widehat{\zeta}-\widehat{\delta}_{S B}\right) \hat{\dot{\varepsilon}}_{12} \hat{\dot{\zeta}}
$$

where the quadratic terms of velocities have been ignored. We can prove that these velocity terms can only produce higher-order small quantities in the final results. If the time rate of momentum in equation (B2) equals the difference of the shear stress in the shear band, then the momentum balance equation is as follows:

$$
\left(\widehat{V}_{S}+\hat{\dot{\varepsilon}}_{12} \operatorname{Re} \widehat{\delta}_{S B}\right) \operatorname{Re} \hat{\dot{\delta}}_{S B}+\left(2 \widehat{\zeta}-\widehat{\delta}_{S B}\right) \hat{\hat{\varepsilon}_{12}} \hat{\zeta}=\frac{1}{\operatorname{Re}}\left[\hat{\tau}_{s}-\hat{\tau}\left(U_{S}\right)\right]
$$

where $\hat{\tau}_{s}$ is the shear yield stress of the material. $\hat{\tau}\left(U_{S}\right)$ is the shear stress in the shear band, which is identified as the linear function of the displacement $U_{S}$ on the shear plane and can be written as follows:

$$
\hat{\tau}\left(U_{S}\right)=\widehat{\tau}_{S}\left(1-\frac{\widehat{U}_{S}}{\widehat{U}_{S c}}\right) \quad 0 \leq \widehat{U}_{S} \leq \widehat{U}_{S c}
$$

where USc is the critical displacement in the shear plane. The dimensionless displacement of the shear plane $\widehat{U}_{S}$ is

$$
\widehat{U}_{S}=2 \int_{0}^{\hat{t}} \hat{\dot{\varepsilon}}_{12}\left(\widehat{\zeta}-\frac{1}{2} \widehat{\delta}_{S B}\right) d \hat{t}
$$

Substituting (B4) into the right-hand side of (B3) and performing a time derivative of (B5), we obtain

$$
\begin{aligned}
& \left(\widehat{V}_{S}+\hat{\dot{\varepsilon}}_{12} \operatorname{Re} \widehat{\delta}_{S B}\right) \operatorname{Re} \hat{\dot{\delta}}_{S B}+\left(2 \widehat{\zeta}-\widehat{\delta}_{S B}\right) \hat{\dot{\varepsilon}_{12}} \hat{\zeta}=\frac{\hat{\zeta}_{S} \widehat{U}_{S}}{\operatorname{Re} \widehat{U}_{S c}} \\
& \hat{\dot{U}}_{S}=\hat{\dot{\varepsilon}}_{12}\left(2 \widehat{\zeta}-\widehat{\delta}_{S B}\right)
\end{aligned}
$$

Since $\hat{\dot{\delta}}_{S B}<<\hat{\dot{\zeta}}$, we may neglect the influence of the time rate of the shear band width. Thus, from (B6), we obtain the following equation on the rigid-plastic boundary position $\hat{\zeta}$ : 


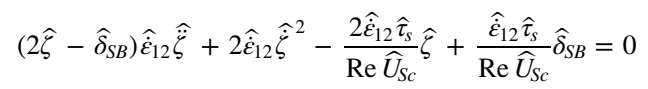

Grady and Kipp (1987) assumed that $\hat{\zeta}$ is a quadratic polynomial function of a time variable. Using this assumption, we have

$$
\widehat{\zeta}=A \hat{t}^{2}+B \hat{t}+C
$$

Substituting (B8) into (B7) and using the initial condition $\widehat{\zeta}(0)=\widehat{U}_{S}(0)=0$, we may easily determine the constants in (B8), $A, B$ and $C$ as follows:

$$
A=\frac{\widehat{\tau}_{S}}{6 \widehat{U}_{S c}}, B=0, C=\frac{\widehat{\delta}_{S B}}{2}-\frac{\widehat{V}_{S}}{4 \hat{\varepsilon}_{12}} .
$$

From (B8) and (B9), we can obtain the expression of the position function of rigid-plastic boundary as follows:

$$
\widehat{\zeta}=\frac{\hat{\tau}_{s}^{2} \hat{t}^{2}}{6 \operatorname{Re} \widehat{E}^{S B}}+\frac{1}{2}\left(\widehat{\delta}_{S B}-\frac{\widehat{V}_{S}}{2 \hat{\dot{\varepsilon}}_{12}}\right)
$$

where $\widehat{E}^{S B}=E^{S B} \operatorname{Re}^{2} / \rho V_{0}^{2} L$ is the dimensionless shear deformation energy and the relationship $\widehat{E}^{S B}=\operatorname{Re} \widehat{\tau}_{s} \widehat{U}_{S c}$ has been introduced in (B10). From (B5) and (B10), we can find

$$
\frac{\hat{\tau}_{s}^{2}}{9 \operatorname{Re} \widehat{E}^{S B}} \hat{t}^{3}-\frac{\widehat{V}_{S}}{2 \hat{\dot{\varepsilon}}_{12}} \hat{t}-\frac{\widehat{U}_{S}}{\hat{\dot{\varepsilon}}_{12}}=0 .
$$

If the terminal time of shear band evolution is denoted by $\hat{t}_{c}$ at which the displacement on the shear plane arrives at its critical displacement, i.e., $\widehat{U}_{S}=\widehat{U}_{S c}$, from (B11), this time $\hat{t}_{c}$ can be determined to be

$$
\hat{t}_{c}=\left[\frac{9 \operatorname{Re} \widehat{E}^{S B 2}}{2 \hat{\dot{\varepsilon}}_{12} \hat{\tau}_{s}^{3}}\left(1+\sqrt{1+\left(\frac{\hat{\tau}_{s} \widehat{V}_{S}}{3 \widehat{E}^{S B}}\right)^{2}}\right)\right]^{\frac{1}{3}} .
$$

In (B10), setting $\hat{t}=\hat{t}_{c}$ and combining the result with (39), we get the critical shear band spacing as follows:

$$
\hat{l}_{S B c}=2 \widehat{\zeta}=\frac{\hat{\tau}_{s}^{2}}{3 \operatorname{Re} \widehat{E}_{m}^{s b}} \hat{t}_{c}^{2}+\widehat{\delta}_{S B c}-\frac{\widehat{V}_{S}}{2 \hat{\dot{\varepsilon}}_{12}},
$$

where $\widehat{E}^{S B}=\widehat{E}_{m}^{S B}, \widehat{\delta}_{S B}=\widehat{\delta}_{S B C}$, and $\hat{l}_{S B}=\hat{l}_{S B c}$ are used. This denotes that, at the end of shear band evolution, the shear deformation energy of the shear band reaches its saturation limit, and the shear band width and spacing reach, respectively, the critical width and spacing.

Consider the heating in shear band from the conversion of plastic work, the energy balance equation in (9) can be rewritten as

$$
\left(1+\frac{\bar{v}_{2} \hat{t}_{c}}{\widehat{\delta}_{S B}}+\frac{R_{c} \operatorname{Re}^{2} \hat{t}_{c}}{2 \widehat{\delta}_{S B}^{2}}\right) \Delta \widehat{T}=\frac{R_{g} \widehat{E}^{S B}}{R_{v} \widehat{\delta}_{S B}}
$$

where approximate relations $\widehat{T}_{, 22}=\Delta \widehat{T} / 2 \widehat{\delta}_{S B}^{2}$ and $\widehat{\sigma}_{12} \widehat{v}_{1,2}=\widehat{E}^{S B} / \widehat{\delta}_{S B}$ have been used. Following the approach of Grady and Kipp (1987), we assume that thermal softening is so strong that the shear stress drops to zero in the shear band when the shear band evolution is fully matured at $\hat{t}=\hat{t}_{c}$. We further assume that the temperature in the shear band arrives at the melting point, i.e., $\Delta \widehat{T}=1$. Thus, the energy balance equation in (B12) is rewritten as

$$
\widehat{E}^{S B}=\frac{R_{v} \widehat{\delta}_{S B c}}{R_{g}}\left(1+\frac{\overline{\hat{v}}_{2} \hat{t}_{c}}{\widehat{\delta}_{S B c}}+\frac{R_{c} \operatorname{Re}^{2} \hat{t}_{c}}{2 \widehat{\delta}_{S B c}^{2}}\right) .
$$

Grady and Kipp (1987) showed that, from the balance condition between heat conduction and momentum diffusion, there must be an optimum shear band width $\hat{\delta}_{S B c}$ corresponding to the maximum softening rate or the minimum localized time $\hat{t}_{c m}$. From relationship (34), we can see that the energy dissipated by shear-localized deformation also reaches the saturation limit $\widehat{E}_{m}^{S B}$. Thus, from Eqn. (41) and the extreme conditions $d \widehat{E}^{S B} / d \widehat{\delta}_{S B}=0$, we obtain the minimum localized time as

$$
\hat{t}_{c m}=\frac{2 R_{v} \widehat{\delta}_{S B c}^{2}}{R_{c}} .
$$

The substitution of Eqn. (42) into Eqn. (41) yields

$$
\widehat{E}_{m}^{s b}=\frac{R_{\nu} \widehat{\delta}_{S B c}}{R_{g}}\left(1+R_{v} \operatorname{Re}^{2}\right),
$$

Ulteriorly, the combination of Eqn. (41) and Eqn. (43) yields the equation satisfied by the critical shear band width $\delta S B c a s$ follows: 


$$
\widehat{\delta}_{S B c}^{6}-\frac{9 \operatorname{Re}_{c} R_{c}^{3}}{2 R_{v} R_{g}^{2} \hat{\varepsilon}_{12} \hat{\tau}_{s}^{3}} \widehat{\delta}_{S B c}^{2}\left(1+\frac{R_{v} \widehat{v}_{2} \widehat{\delta}_{S B c}}{\operatorname{Re}_{c} R_{c}}\right)^{2}-\frac{\operatorname{Re}_{c} R_{c}^{3} \widehat{V}_{S}^{2}}{32 \hat{\varepsilon}_{12} \widehat{\tau}_{v} R_{v}^{3}}=0 .
$$

We may prove that the terms related to v2 and VS are small quantities. Therefore, neglecting the influences of the two terms on SSBcand solving this equation, we obtain the critical width of shear band as

$$
\widehat{\delta}_{S B c}=\left(\frac{9 \operatorname{Re}_{c} R_{c}^{3}}{2 R_{v} R_{g}^{2} \hat{\varepsilon}_{12} \hat{\tau}_{s}^{3}}\right)^{\frac{1}{4}} .
$$

\section{Appendix C. Supplementary material}

Supplementary data associated with this article can be found in the online version at http://dx.doi.org/10.1016/j.jmps.2016.07. 023.

\section{References}

Atlati, S., Haddag, B., Nouari, M., Zenasni, M., 2011. Analysis of a new segmentation intensity ratio "SIR" to characterize the chip segmentation process in machining ductile metals. Int. J. Mach. Tools Manuf. 51, 687-700.

Atkins, A.G., 2003. Modelling metal cutting using modern ductile fracture mechanics: quantitative explanations for some longstanding problems. Int. J. Mech. Sci. 45, $373-396$.

Bai, Y.L., 1982. Thermo-plastic instability in simple shear. J. Mech. Phys. Solids 36, 195-207.

Bäker, M., Röosler, J., Siemers, C., 2002. A finite element model of high speed metal cutting with adiabatic shearing. Comput. Struct. 80, $495-513$.

Barry, J., Byrne, G., 2002. The mechanisms of chip formation in machining hardened steels. J. Manuf. Sci. Eng. 124, 528-535.

Barry, J., Byrne, G., Lennon, D., 2001. Observations on chip formation and acoustic emission in machining Ti6Al4V alloy. Int. J. Mach. Tools Manuf. 41, 1055-1070. Bisacre, F.F.P., Bisacre, G.H., 1947. The life of carbide-tipped turning tools. Pro. Int. Mech. Eng. 157, 452-469.

Burns, T.J., Davies, M.A., 1997. Nonlinear dynamics model for chip segmentation in machining. Phys. Rev. Lett. 79, 447-450.

Burns, J., Davies, M.A., 2002. On repeated adiabatic shear band formation during high-speed machining. Int. J. Plast. 18, 487-506.

Chen, G., Ren, C.G., Yang, X., Jin, X.M., Guo, T., 2011. Finite element simulation of high-speed machining of titanium alloy (Ti-6Al-4V) based on ductile failure model. Int. J. Adv. Manuf. Technol. 56, 1027-1038.

Cotterell, M., Byrne, G., 2008. Dynamics of chip formation during orthogonal cutting of titanium alloy Ti-6Al-4V. CIRP Ann.-Manuf. Technol. 57, 93-96.

Elbestawi, M.A., Srivastava, A.K., El-Wardany, T.I., 1996. A model for chip formation during machining of hardened steel. CIRP Ann. 45, 71-76.

Enahoro, H., E., Oxley, P.L.B., 1966. Flow along Tool-Chip interface in orthogonal metal cutting. J. Mech. Eng., Sci. 8, 36-41.

Fang, N., 2003. Slip-Line modeling of machining with a rounded-edge tool. Part I: new model and theory. J. Mech. Phys. Solids 51, 715-742.

Gente, A., Hoffmeister, H.-W., 2001. Chip formation in machining Ti6Al4V at extremely high cutting speeds. Ann. CIRP 50 (1), $49-52$.

Grady, D.E., Kipp, M.E., 1987. The growth of unstable thermoplastic shear with application to steady-wave shock compression in solids. J. Mech. Phys. Solids 35, 95-119.

Gioia, G., Ortiz, M., 1996. The two-dimensional structure of dynamic boundary layers and shear bands in thermoviscoplastic solids. J. Mech. Phys. Solids 44, 251-292.

Gu, Liyao, Wang, Minjie, Duan, Chunzheng, 2013. On adiabatic shear localized fracture during serrated chip evolution in high speed machining of hardened AISI 1045 steel. Int. J. Mech. Sci. 75, 288-298.

Johnson, G.R., Cook, W.H., 1985. Fracture characteristic of three metals subjected to various strains, strain rates, temperatures and pressures. Eng. Fract. Mech. 21, 31.

Komanduri, R., Hou, Z.B., 2001. Thermal modeling of the metal cutting process -Part III: temperature rise distribution due to the combined effects of shear plane heat source and the tool-chip interface frictional heat source. Int. J. Mech. Sci. 43, 89-107.

Lee, W.S., Lin, C.F., 1998. High-temperature deformation behavior of Ti6Al4V alloy evaluated by high strain-rate compression tests. J. Mater. Process. Technol. 75, 127-136.

Ma, W., Li, X.W., Dai, L.H., Ling, Z., 2012. Instability criterion of materials in combined stress states and its application to orthogonal cutting process. Int. J. Plast. 30-31, 18-40.

Merchant, M.E., 1945. Mechanics of the metal cutting process. I. Orthogonal cutting and a type 2 chip. J. Appl. Phys. 16, $267-275$.

Meyers, M.A., Nesterenko, V.F., LaSalvia, J.C., Xue, Q., 2001. Shear localization in dynamic deformation of materials: microstructural evolution and selforganization. Mater. Sci. Eng. A 317, 204-225.

Molinari, A., 1997. Collective behavior and spacing of adiabatic shear bands. J. Mech. Phys. Solids 45, 1551-1575.

Molinari, A., Musquar, C., Sutter, G., 2002. Adiabatic shear banding in high speed machining of Ti-6Al-4V: experiments and modeling. Int. J. Plast. 18, 443-459.

Molinari, A., Soldani, X., Miguélez, M.H., 2013. Adiabatic shear banding and scaling laws in chip formation with application to cutting of Ti-6Al-4V. J. Mech. Phys. Solids 61, 2331-2359.

Oxley, P. L. B. and Welsh, M. J. M., 1963. Calculating the shear angle in orthogonal metal cutting from fundamental stress, strain-rate properties of the work material. In: Proceedings 4th International Machine Tool Design and Research Conference. Pergamon, Oxford, pp. 73-86.

Oxley, P.L.B., 1989. The mechanics of machining: an analytical approach to assessing machinability. Halsted Press: A division of John Wiley and Sons Limited, New York.

Palmer, W.B., Oxley, P.L.B., 1959. Mechanics of metal cutting. Proc. Inst. Mech. Eng. 173, 623-645.

Piispanen, V., 1948. Theory of formation of metal chips. J. Appl. Phys. 19, 876-881.

Poulachon, G., Moisan, A., 1998. A contribution to the study of the cutting mechanisms during high speed machining of hardened steel. CIRP Ann., 47 1, 73-76. Roth, R.N., 1969. Analysis of Flow Round Tool Cutting Edge (PhD Thesis). University of New South Wales, Sydney, Australia.

Sekhon, G.S., Chenot, J.L., 1993. Numerical simulation of continuous chip formation during non-steady orthogonal cutting. Eng. Comput. 10 , 31-48.

Shaw, M.C., 1984. Metal Cutting Principles. Clarendon Press, Oxford.

Shaw M.C., Janakiram M., Vyas A., 1991. The role of fracture in metal cutting chip formation. SME, NSF Grantees Conference, Austin, Texas, pp. 359-366.

Stevenson, M.G., Oxley, P.L.B., 1970-1971. An experimental investigation of the influence of strain-rate and temperature on the flow stress properties of a low carbon steel using a machining test. Proc. Inst. Mech. Eng. 185, 741-754.

Sutter, G., Faure, L., Molinari, A., Delime, A., Dudzinski, D., 1997. Experimental analysis of the cutting process and chip formation at high speed machining. J. Phys. IV $7,33-38$. 
Sutter, G., List, G., 2013. Very high speed cutting of Ti-6Al-4V titanium alloy-change in morphology and mechanism of chip formation. Int. J. Mach. Tools Manuf. $66,37-43$.

Taylor, F.W., 1906. On the Art of Cutting Metals. Trans. Am. Soc. Mech. Eng. XXVIII, 31-350.

Wang, H., To, S., Chan, C.Y., Cheung, C.F., Lee, W.B., 2011. Dynamic modelling of shear band formation and tool-tip vibration in ultra-precision diamond turning. Int. J. Mach. Tools Manuf. 51, 512-519.

Wright, T.W., Ockendon, H., 1996. A scale law for the effect of inertia on the formation of adiabatic shear bands. Int. J. Plast. $12,927-934$.

Xue, Q., Meyers, M.A., Nesterenko, V.F., 2002. Self-organization of shear bands in titanium and Ti-6Al-4V alloy. Acta Materialia 50, 575-596.

Xue, Q., Meyers, M.A., Nesterenko, V.F., 2004. Self organization of shear bands in stainless steel. Mater. Sci. Eng. $384,35-46$.

Ye, G.G., Xue, S.F., Ma, W., Jiang, M.Q., Ling, Z., Tong, X.H., Dai, L.H., 2012. Cutting AISI 1045 steel at very high speeds. Int. J. Mach. Tools Manuf. 56, 1-9.

Ye, G.G., Xue, S.F., Jiang, M.Q., Tong, X.H., Dai, L.H., 2013. Modeling periodic adiabatic shear band evolution during high speed machining Ti-6Al-4V alloy. Int. J. Plast. 40, 39-55. 